JOURNAL OF

FUNCTION SPACES AND APPLICATIONS

Volume 4, Number 2 (2006), 193-220
(C) 2006, Scientific Horizon http://www.jfsa.net

\title{
Littlewood-Paley characterization for Campanato spaces
}

\author{
Azzeddine El Baraka \\ (Communicated by Hans Triebel)
}

2000 Mathematics Subject Classification. 42B25.

Keywords and phrases. Campanato spaces, BMO and bmo, Theorems of Littelwood Paley type.

\footnotetext{
Abstract. The Littlewood-Paley characterization for the local approximation Campanato spaces $L_{p}^{\alpha}$ is well known in the cases $\alpha \geq 0$ and $\alpha=-\frac{n}{p}$. We give in this paper a characterization of such a type for $L_{2}^{\alpha}$ spaces (and for MorreyCampanato spaces $\mathcal{L}^{2, \lambda}$ ) for any $\alpha \geq-\frac{n}{2}$. These spaces contain as spacial cases the well known spaces $B M O$ of John and Nirenberg and its local version bmo.
}

\section{Introduction and results}

In this paper, we make use of a partition of Littlewood-Paley type to get a dyadic characterization for the spaces $B M O, b m o$ and more generally for Campanato spaces $\mathcal{L}^{2, \lambda}$ modulo polynomials and their local versions $L_{2}^{\alpha}$. In this direction we mention the following classical characterization of $B M O$ and bmo with the aid of Triebel-Lizorkin spaces $F_{p, q}^{s}\left(\mathbf{R}^{n}\right): B M O=\dot{F}_{\infty, 2}^{0}$, bmo $=F_{\infty, 2}^{0}$ and $I^{s}(B M O)=\dot{F}_{\infty, 2}^{s}$, where $I^{s}=\mathcal{F}^{-1}\left(|\cdot|^{-s} \mathcal{F}\right)$ is the Riesz 
potential operator, cf. [15, chapters 2 and 5]. The spaces $I^{s}(B M O)$ were studied by Strichartz [14].

If we denote $L_{p}^{\alpha}$ the local approximation Campanato spaces defined for instance in the book [16, Definition 1.7.2 (5)] for $\alpha \geq-\frac{n}{p}$ and $1 \leq p<+\infty$, then we recall that $L_{p}^{\alpha}=\mathcal{C}^{\alpha}$ for any $\alpha>0, L_{p}^{-n / p}=L^{p}$ and $L_{p}^{0}=b m o$, cf. [4], [12], [16] and [17] for the proof and more references. In the case $-\frac{n}{p}<\alpha<0$, the spaces $L_{p}^{\alpha}$ coincide with Morrey spaces (introduced to complement the scale of $L^{p}$ spaces) which are themselves equal to Campanato spaces $\mathcal{L}^{p, \lambda}, \lambda=n+\alpha p$ considered in this work, cf. Definition 5. The main result of this paper is the dyadic characterization for $L_{2}^{\alpha}$ spaces and $I^{s}\left(L_{2}^{\alpha}\right)$ which is of interest essentially in the case $-\frac{n}{2}<\alpha<0$. By the way, we mention that this characterization is not true for the general spaces $L_{p}^{\alpha}, 1 \leq p<+\infty, p \neq 2$, cf. Remark 11.

We start by recalling the definition of the spaces $\mathcal{L}^{p, \lambda, s}\left(\mathbf{R}^{n}\right)$ and their homogeneous counterparts $\dot{\mathcal{L}}^{p, \lambda, s}\left(\mathbf{R}^{n}\right)$ which involve expressions with balls (or cubes) of $\mathbf{R}^{n}$ and a resolution of Littlewood-Paley. These spaces were introduced in the previous papers [9] and [5] in order to solve a conjecture of Hans Triebel regarding an isomorphism theorem for elliptic operators in $B M O$ and bmo spaces, cf. [15, section 4.3.4].

Next, we show that for any $s \in \mathbf{R}$ and $0 \leq \lambda<n+2$, the space $\dot{\mathcal{L}}^{2, \lambda, s}$ coincides with the Riesz-potential space $I^{s}\left(\mathcal{L}^{2, \lambda}\right)$ built on Campanato space $\mathcal{L}^{2, \lambda}$ modulo polynomials (Theorem 10). In particular, for $s=0$ we get the dyadic characterization for Campanato spaces $\mathcal{L}^{2, \lambda}$ modulo polynomials and their local versions $L_{2}^{\alpha}$, cf. Proposition 8.

To prove these results we follow up the characterization of $B M O$ given in $[1]$.

To make this paper self contained we recall the definition of the spaces introduced in previous papers. For this we need a Littlewood-Paley partition of unity. We denote $x \in \mathbf{R}^{n}$ and $\xi$ its dual variable. Let $\varphi \in C_{0}^{\infty}\left(\mathbf{R}^{n}\right), \varphi \geq$ 0 and $\varphi$ equal to 1 for $|\xi| \leq 1,0$ for $|\xi| \geq 2$; and set $\theta(\xi)=\varphi(\xi)-\varphi(2 \xi)$, $\left(\operatorname{supp} \theta \subset\left\{\frac{1}{2} \leq|\xi| \leq 2\right\}\right)$. For $j \in \mathbf{Z}$ we set

$$
\dot{\Delta}_{j} u=\theta\left(2^{-j} D_{x}\right) u
$$

and denoting $\psi_{j}(\xi)=\sum_{k \leq j} \theta\left(2^{-k} \xi\right)\left(=\varphi\left(2^{-j} \xi\right)\right.$ for $\left.\xi \neq 0\right)$ we set

$$
\dot{S}_{j} u=\psi_{j}\left(D_{x}\right) u
$$


If $j \geq 1$ we set also

$$
\Delta_{j} u=\dot{\Delta}_{j} u, S_{j} u=\varphi\left(2^{-j} D_{x}\right) u
$$

and

$$
S_{0} u=\Delta_{0} u=\varphi\left(D_{x}\right) u
$$

Remark 1. We have $\varphi(\xi)+\sum_{k \geq 1} \theta\left(2^{-k} \xi\right)=1$ for any $\xi \in \mathbf{R}^{n}$, thus the nonhomogeneous partition of $u \in \mathcal{S}^{\prime}\left(\mathbf{R}^{n}\right)$ is given by the formula

$$
u=\sum_{k \geq 0} \Delta_{k} u=\Delta_{0} u+\sum_{k \geq 1} \Delta_{k} u=S_{j} u+\sum_{k \geq j+1} \Delta_{k} u \text { for } j \in \mathbf{N} .
$$

Set $f(\xi)=\sum_{k \in \mathbf{Z}} \theta\left(2^{-k} \xi\right)$. For each fixed $\xi \neq 0, f(\xi)$ contains at most two non-vanishing terms, we deduce $f(\xi)=1$ for any $|\xi| \geq 2$. We note that $f\left(2^{-j} \xi\right)=f(\xi)$ for any $j \in \mathbf{Z}$, and choosing $j$ larger and larger, we obtain $f(\xi)=1$ for any $\xi \neq 0$. Then, for $u \in \mathcal{S}^{\prime}\left(\mathbf{R}^{n}\right)$, with $0 \notin \operatorname{supp} \mathcal{F} u$, the homogeneous partition of $u$ is given by the formula

$$
u=\sum_{k \in \mathbf{Z}} \dot{\Delta}_{k} u=\dot{S}_{j} u+\sum_{k \geq j+1} \dot{\Delta}_{k} u \text { for } j \in \mathbf{Z} .
$$

Now we recall the definition of the nonhomogeneous spaces introduced in [5] and [6].

Definition 2. Let $s \in \mathbf{R}, \lambda \geq 0$ and $1 \leq p<+\infty$. The space $\mathcal{L}^{p, \lambda, s}\left(\mathbf{R}^{n}\right)$ denotes the set of all tempered distributions $u \in \mathcal{S}^{\prime}\left(\mathbf{R}^{n}\right)$ such that

$$
\|u\|_{\mathcal{L}^{p, \lambda, s}\left(\mathbf{R}^{n}\right)}=\left\{\sup _{B} \frac{1}{|B|^{\frac{\lambda}{n}}} \sum_{j \geq J^{+}} 2^{j p s}\left\|\Delta_{j} u\right\|_{L^{p}(B)}^{p}\right\}^{\frac{1}{p}}<+\infty
$$

where $J^{+}=\max (J, 0),|B|$ is the measure of $B$ and the supremum is taken over all $J \in \mathbf{Z}$ and all balls $B$ of $\mathbf{R}^{n}$ of radius $2^{-J}$.

The space $\mathcal{L}^{p, \lambda, s}\left(\mathbf{R}^{n}\right)$ equipped with the norm (1) is a Banach space.

To give the homogeneous counterpart of the spaces $\mathcal{L}^{p, \lambda, s}\left(\mathbf{R}^{n}\right)$, we recall the notation of $[15$, chapter 5]. Let

$$
Z\left(\mathbf{R}^{n}\right)=\left\{\varphi \in \mathcal{S}\left(\mathbf{R}^{n}\right) ;\left(D^{\alpha} \mathcal{F} \varphi\right)(0)=0 \text { for every multi-index } \alpha\right\}
$$

$Z\left(\mathbf{R}^{n}\right)$ is considered as a subspace of $\mathcal{S}\left(\mathbf{R}^{n}\right)$ with the same topology, and $Z^{\prime}\left(\mathbf{R}^{n}\right)$ is the topological dual of $Z\left(\mathbf{R}^{n}\right)$. We may identify $Z^{\prime}\left(\mathbf{R}^{n}\right)$ and 
$\mathcal{S}^{\prime}\left(\mathbf{R}^{n}\right) / \mathcal{P}$, where $\mathcal{P}$ is the set of all polynomials of $\mathbf{R}^{n}$ with complex coefficients, ie. $Z^{\prime}\left(\mathbf{R}^{n}\right)$ is interpreted as $\mathcal{S}^{\prime}\left(\mathbf{R}^{n}\right)$ modulo polynomials.

Definition 3. Let $s \in \mathbf{R}, \lambda \geq 0$ and $1 \leq p<+\infty$. The dotted space $\dot{\mathcal{L}}^{p, \lambda, s}\left(\mathbf{R}^{n}\right)$ denotes the set of all $u \in Z^{\prime}\left(\mathbf{R}^{n}\right)$ such that

$$
\|u\|_{\dot{\mathcal{L}}^{p, \lambda, s}\left(\mathbf{R}^{n}\right)}=\left\{\sup _{B} \frac{1}{|B|^{\frac{\lambda}{n}}} \sum_{j \geq J} 2^{j p s}\left\|\dot{\Delta}_{j} u\right\|_{L^{p}(B)}^{p}\right\}^{\frac{1}{p}}<+\infty
$$

where the supremum is taken over all $J \in \mathbf{Z}$ and all balls $B$ of $\mathbf{R}^{n}$ of radius $2^{-J}$.

If $P$ is a polynomial of $\mathcal{P}$ and $u \in \mathcal{S}^{\prime}\left(\mathbf{R}^{n}\right)$, it follows immediatly that

$$
\|u+P\|_{\dot{\mathcal{L}}^{p, \lambda, s}\left(\mathbf{R}^{n}\right)}=\|u\|_{\dot{\mathcal{L}}^{p, \lambda, s}\left(\mathbf{R}^{n}\right)}
$$

This shows that the norm (2) is well defined. Further, the space $\dot{\mathcal{L}}^{p, \lambda, s}\left(\mathbf{R}^{n}\right)$ equipped with this norm is a Banach space.

Remark 4. The supremum in expressions (1) and (2) can be taken over all $J \in \mathbf{Z}$ and all cubes $B$ of $\mathbf{R}^{n}$ of length side $2^{-J}$.

We give in [5], [6] and [7] some properties of these spaces.

In order to compare $\dot{\mathcal{L}}^{p, \lambda, 0}\left(\mathbf{R}^{n}\right)$ with Campanato space $\mathcal{L}^{p, \lambda}\left(\mathbf{R}^{n}\right)$ we must interpret the latter as a subspace of $Z^{\prime}\left(\mathbf{R}^{n}\right)$.

Definition 5 (Campanato spaces). Let $\lambda \geq 0$ and $1 \leq p<+\infty$.

(i) The space $\mathcal{L}^{p, \lambda}\left(\mathbf{R}^{n}\right)$ denotes the set of all functions $u \in L_{l o c}^{p}\left(\mathbf{R}^{n}\right)$ such that

$$
\|u\|_{\mathcal{L}^{p, \lambda}\left(\mathbf{R}^{n}\right)}=\left\{\sup _{B} \frac{1}{|B|^{\frac{\lambda}{n}}} \int_{B}\left|u-m_{B} u\right|^{p} d x\right\}^{\frac{1}{p}}<+\infty
$$

where $m_{B} u=\frac{1}{|B|} \int_{B} u(y) d y$ is the mean value of $u$ and the supremum is taken over all balls $B$ of $\mathbf{R}^{n}$.

We note that $\mathcal{L}^{p, \lambda}\left(\mathbf{R}^{n}\right)$ is a Banach space modulo constants equal to $\{0\}$ if $\lambda>n+p$.

(ii) For $0 \leq \lambda<n+p$ we define the space $\dot{\mathcal{L}}^{p, \lambda}\left(\mathbf{R}^{n}\right)$ as the set of all equivalence classes $U$ modulo $\mathcal{P}$ of elements of $\mathcal{L}^{p, \lambda}\left(\mathbf{R}^{n}\right)$, equipped with the norm $\|U\|_{\mathcal{L}^{p, \lambda}\left(\mathbf{R}^{n}\right)}=\|u\|_{\mathcal{L}^{p, \lambda}\left(\mathbf{R}^{n}\right)}$ where $u$ is the unique (modulo constants) element of $\mathcal{L}^{p, \lambda}\left(\mathbf{R}^{n}\right)$ belonging to the class $U$. Througout this paper we identify $U$ with $u$. 
We denote $B M O=\dot{\mathcal{L}}^{1, n}\left(\mathbf{R}^{n}\right)$.

Now we define the local approximation Campanato spaces $\mathcal{L}_{p}^{\alpha}\left(\mathbf{R}^{n}\right)$ which are connected to $\mathcal{L}^{p, \lambda}\left(\mathbf{R}^{n}\right)$. We follow Wallin [17] and Triebel [16, Definition 1.7.2(5)].

Definition 6 (Local approximation spaces). Let $1 \leq p<+\infty, \alpha \geq-\frac{n}{p}$ and $N=\max (-1,[\alpha]),[\alpha]$ is the integer part of $\alpha$. We say $u \in \mathcal{L}_{p}^{\alpha}\left(\mathbf{R}^{n}\right)$ if and only if $u \in L_{l o c}^{p}\left(\mathbf{R}^{n}\right)$ and for some constant $M=M(u)$, for every cube $B$ of length side $\delta$, there exists a polynomial $P_{B}$ of degree $\leq N\left(P_{B}=0\right.$ if $\left.N=-1\right)$ such that

$$
\left\{\frac{1}{|B|^{1+\alpha p / n}} \int_{B}\left|u(x)-P_{B}\right|^{p} d x\right\}^{\frac{1}{p}} \leq M \text { if } 0<\delta \leq 1
$$

and

$$
\left\{\frac{1}{|B|} \int_{B}|u(x)|^{p} d x\right\}^{\frac{1}{p}} \leq M \text { if } \delta=1
$$

We denote by $\|u\|_{\mathcal{L}_{p}^{\alpha}}$ the infimum of the constants $M$ as above.

We observe that (4) implies (4) for $\delta>1$ which implies (3) for $\delta>1$ with $P_{B}=0$.

We denote $b m o=\mathcal{L}_{1}^{0}\left(\mathbf{R}^{n}\right)$ and $l^{p, \lambda}\left(\mathbf{R}^{n}\right)=\mathcal{L}_{p}^{\frac{\lambda-n}{p}}\left(\mathbf{R}^{n}\right)$.

Remark 7. (i) The space $\mathcal{L}^{p, \lambda}\left(\mathbf{R}^{n}\right), \lambda=n+\alpha p$, coincides with the space defined by requiring (3) for $\delta>0$ and omitting (4), cf. Lemma 17.

(ii) In the case $0 \leq \lambda<n$ the space $l^{p, \lambda}\left(\mathbf{R}^{n}\right)\left(=\mathcal{L}_{p}^{\frac{\lambda-n}{p}}\left(\mathbf{R}^{n}\right)\right)$ coincides with Morrey space $L^{p, \lambda}\left(\mathbf{R}^{n}\right)$ (itself equal to $\mathcal{L}^{p, \lambda}\left(\mathbf{R}^{n}\right)$ ) defined as the set of all $u \in L_{l o c}^{p}\left(\mathbf{R}^{n}\right)$ such that

$$
\|u\|_{L^{p, \lambda}\left(\mathbf{R}^{n}\right)}=\left\{\sup _{B} \frac{1}{|B|^{\frac{\lambda}{n}}} \int_{B}|u(x)|^{p} d x\right\}^{\frac{1}{p}}<+\infty
$$

the sup being over all balls of $\mathbf{R}^{n}$.

The following proposition gives the dyadic characterization of $\dot{\mathcal{L}}^{2, \lambda}\left(\mathbf{R}^{n}\right)$ and $l^{2, \lambda}\left(\mathbf{R}^{n}\right)$.

Proposition 8. Let $0 \leq \lambda<n+2$.

(i) The space $\dot{\mathcal{L}}^{2, \lambda, 0}\left(\mathbf{R}^{n}\right)$ coincides algebraically and topologically with Campanato space $\dot{\mathcal{L}}^{2, \lambda}\left(\mathbf{R}^{n}\right)$. In particular, $\dot{\mathcal{L}}^{2, n, 0}\left(\mathbf{R}^{n}\right) \equiv B M O$. 
(ii) The space $\mathcal{L}^{2, \lambda, 0}\left(\mathbf{R}^{n}\right)$ coincides algebraically and topologically with the space $l^{2, \lambda}\left(\mathbf{R}^{n}\right)$. In particular, $\mathcal{L}^{2, n, 0}\left(\mathbf{R}^{n}\right) \equiv$ bmo.

This proposition allow us to deduce the link between the discrete scale built on the space $\dot{\mathcal{L}}^{2, \lambda}\left(\mathbf{R}^{n}\right)$ and the continuous one:

Corollary 9. Let $0 \leq \lambda<n+2$ and $m \in \mathbf{N}$.

(i) $\dot{\mathcal{L}}^{2, \lambda, m}\left(\mathbf{R}^{n}\right) \hookrightarrow \dot{\mathcal{H}}^{2, \lambda, m}:=\left\{u \in \dot{\mathcal{L}}^{2, \lambda}\left(\mathbf{R}^{n}\right) ; D^{\alpha} u \in \dot{\mathcal{L}}^{2, \lambda}\left(\mathbf{R}^{n}\right),|\alpha| \leq m\right\}$.

(ii) $\dot{\mathcal{H}}^{2, \lambda, m} \cap \dot{H}^{m+\lambda / 2}\left(\mathbf{R}^{n}\right) \hookrightarrow \dot{\mathcal{L}}^{2, \lambda, m}\left(\mathbf{R}^{n}\right)$, here $\dot{H}^{m+\lambda / 2}\left(\mathbf{R}^{n}\right)$ is the classical homogeneous Sobolev space. Therefore,

$$
\dot{\mathcal{H}}^{2, \lambda, m} \bigcap \dot{H}^{m+\lambda / 2}\left(\mathbf{R}^{n}\right) \equiv \dot{\mathcal{L}}^{2, \lambda, m}\left(\mathbf{R}^{n}\right) \bigcap \dot{H}^{m+\lambda / 2}\left(\mathbf{R}^{n}\right)
$$

To state a more general result, we introduce the Riesz potential operator

$$
I^{s} f=\mathcal{F}^{-1}\left\{|\cdot|^{-s} \mathcal{F} f\right\}, \quad f \in Z^{\prime}\left(\mathbf{R}^{n}\right) \text { and } s \in \mathbf{R}
$$

Theorem 10. Let $s \in \mathbf{R}$ and $0 \leq \lambda<n+2$.

(i) The space $\dot{\mathcal{L}}^{2, \lambda, s}\left(\mathbf{R}^{n}\right)$ coincides algebraically and topologically with the space $I^{s}\left(\dot{\mathcal{L}}\left(\mathbf{R}^{n}\right)\right)$ image of $\dot{\mathcal{L}}\left(\mathbf{R}^{n}\right)$ under $I^{s}$.

(ii) The space $\mathcal{L}^{2, \lambda, s}\left(\mathbf{R}^{n}\right)$ coincides algebraically and topologically with the space $I^{s}\left(l^{2, \lambda}\left(\mathbf{R}^{n}\right)\right)$.

Remark 11. We mention that these results are not true in general for $p \neq 2$. G. Bourdaud has noted that $\dot{\mathcal{L}}^{p, 0,0}=\dot{B}_{p, p}^{0}$ for any $1 \leq p<+\infty$, and it is classical that $\dot{\mathcal{L}}^{p, 0}=L^{p}$, therefore the space $\dot{\mathcal{L}}^{p, \lambda, 0}$ does not coincide in general with Campanato space $\dot{\mathcal{L}}^{p, \lambda}$. Nevertheless, we give some injections between $\dot{\mathcal{L}}^{p, \lambda}$ and $\dot{\mathcal{L}}^{p, \lambda, 0}$ in Propositions 21, 22 and 26.

We start with some helpful lemmas needed in the further considerations.

\section{Some preliminary lemmas}

Througout this paper, $C, C_{M} \ldots$ denote positive constants whose values may change from line to line. First of all, we recall the following lemmas proved in [6].

Lemma 12. Let $s \in \mathbf{R}, \lambda \geq 0$ and $1 \leq q \leq p<+\infty$. We have the following continuous embedding

$$
F_{p, q}^{s+\frac{n}{p}}\left(\mathbf{R}^{n}\right) \hookrightarrow \mathcal{L}^{p, \lambda, s-\frac{\lambda-n}{p}}\left(\mathbf{R}^{n}\right)
$$


This embedding remains true for the dotted spaces $\dot{F}$ and $\dot{\mathcal{L}}$.

Lemma 13. For any $u \in \mathcal{S}^{\prime}\left(\mathbf{R}^{n}\right)$ we have the decomposition

$$
u=\sum_{j \in \mathbf{Z}} \dot{\Delta}_{j} u \text { in } Z^{\prime}\left(\mathbf{R}^{n}\right) .
$$

Lemma 14. Let $1 \leq p<+\infty$, and $A$ a real $<0$. If $\left(a_{j \nu}\right)_{j, \nu}$ is a sequence of positive real numbers satisfying $\left(a_{j \nu}\right)_{j} \in l^{p}$ for any $\nu \geq 1$, then there exists a constant $C>0$ such that

$$
\sum_{j \geq 1}\left(\sum_{\nu \geq 1} 2^{\nu A} a_{j \nu}\right)^{p} \leq C \sup _{\nu \geq 1} \sum_{j \geq 1} a_{j \nu}^{p}
$$

Lemma 15. Let $1 \leq p<+\infty$, and $s \in \mathbf{R}$. Further, let $\lambda$ and $\alpha \geq 0$. Then we have the following continuous embedding

$$
\mathcal{L}^{p, \lambda, s+\frac{\alpha}{p}}\left(\mathbf{R}^{n}\right) \hookrightarrow \mathcal{L}^{p, \lambda+\alpha, s}\left(\mathbf{R}^{n}\right)
$$

We have the same result for the dotted spaces $\dot{\mathcal{L}}$.

The following lemma is proved in [9] and [8].

Lemma 16. For any integer $M>0$, there exists a constant $C_{M}>0$ such that for any $J \in \mathbf{Z}, x_{0} \in \mathbf{R}^{n}$, for any ball $B$ centered at $x_{0}$ with radius $2^{-J}$, for any $l \in \mathbf{Z}, k \in \mathbf{N}$ and $u \in W_{l o c}^{k, p}\left(\mathbf{R}^{n}\right) \cap L^{p}\left(\mathbf{R}^{n}\right), 1 \leq p \leq+\infty$

$$
\begin{aligned}
\left\|\dot{\Delta}_{l} u\right\|_{L^{p}(B)} \leq & C_{k} 2^{k J} 2^{-k l} \sup _{|\alpha| \leq k}\left\|D_{x}^{\alpha} u\right\|_{L^{p}(3 B)} \\
& +C_{M} \sum_{\nu \geq-J+1} 2^{-(\nu+l) M}\|u\|_{L^{p}\left(F_{\nu}\right)}
\end{aligned}
$$

and

(6) $\left\|\dot{\Delta}_{l} u\right\|_{L^{p}(B)} \leq C_{k} 2^{-k l} \sup _{|\alpha| \leq k}\left\|D_{x}^{\alpha} u\right\|_{L^{p}(3 B)}+C_{M} 2^{-(l-J) M}\|u\|_{L^{p}\left(\mathbf{R}^{n}\right)}$

hold with $F_{\nu}=\left\{x \in \mathbf{R}^{n}: 2^{\nu} \leq\left|x-x_{0}\right| \leq 2^{\nu+1}\right\}$.

Lemma 17. Let $\lambda \geq 0$ and $1 \leq p<+\infty$. Let $u \in L_{l o c}^{p}\left(\mathbf{R}^{n}\right)$.

If for each ball $B$ of $\mathbf{R}^{n}$, there exists a real $c_{B}$ such that

$$
\sup _{B} \frac{1}{|B|^{\frac{\lambda}{n}}} \int_{B}\left|u-c_{B}\right|^{p} d x=C<+\infty
$$

then $u \in \mathcal{L}^{p, \lambda}\left(\mathbf{R}^{n}\right)$ and $\|u\| \leq 2 C^{\frac{1}{p}}$. 
Proof. We write

$\left(\int_{B}\left|u-m_{B} u\right|^{p} d x\right)^{\frac{1}{p}} \leq\left(\int_{B}\left|u-c_{B}\right|^{p} d x\right)^{\frac{1}{p}}+\left(\int_{B}\left|c_{B}-m_{B} u\right|^{p} d x\right)^{\frac{1}{p}}$

Now

$$
\begin{aligned}
\left|c_{B}-m_{B} u\right| & \leq \frac{1}{|B|} \int_{B}\left|u-c_{B}\right| d x \\
& \leq\left(\frac{1}{|B|} \int_{B}\left|u-c_{B}\right|^{p} d x\right)^{\frac{1}{p}} \\
& \leq\left(C|B|^{\frac{\lambda}{n}-1}\right)^{\frac{1}{p}} .
\end{aligned}
$$

Lemma 18. Let $1 \leq p<+\infty, 0 \leq \lambda<n+p$, and $\alpha=\left\{\begin{array}{l}0 \text { if } 0 \leq \lambda \leq n \\ 1 \text { if } \lambda>n\end{array}\right.$.

There exists $C_{n}>0$ such that

$$
m_{2^{j} B}|u| \leq\left|m_{B} u\right|+C_{n}(j+1)\left|2^{\alpha j} B\right|^{\frac{1}{p}\left(\frac{\lambda}{n}-1\right)}\|u\|_{\dot{\mathcal{L}}^{p, \lambda}\left(\mathbf{R}^{n}\right)}
$$

for all balls $B$ of $\mathbf{R}^{n}, j$ integer $\geq 1$ and $u \in \dot{\mathcal{L}}^{p, \lambda}\left(\mathbf{R}^{n}\right)$.

Remark 19. In the case $\lambda>n$, we may remove the term $j+1$ from the right hand side of the last inequality as we will see in the proof.

Proof.

$$
\begin{aligned}
m_{B}|u| & =\frac{1}{|B|} \int_{B}|u(y)| d y \\
& \leq \frac{1}{|B|} \int_{B}\left|u(y)-m_{B} u\right| d y+\left|m_{B} u\right| \\
& \leq|B|^{\frac{1}{p}\left(\frac{\lambda}{n}-1\right)}\left\{\frac{1}{|B|^{\frac{\lambda}{n}}} \int_{B}\left|u(y)-m_{B} u\right|^{p} d y\right\}^{\frac{1}{p}}+\left|m_{B} u\right| \\
& \leq|B|^{\frac{1}{p}\left(\frac{\lambda}{n}-1\right)}\|u\|_{\mathcal{L}^{p, \lambda}\left(\mathbf{R}^{n}\right)}+\left|m_{B} u\right|
\end{aligned}
$$

Replacing $B$ by $2^{j} B$ yields

$$
m_{2^{j} B}|u|-\left|2^{j} B\right|^{\frac{1}{p}\left(\frac{\lambda}{n}-1\right)}\|u\|_{\mathcal{L}^{p, \lambda}\left(\mathbf{R}^{n}\right)} \leq\left|m_{2^{j} B} u\right|
$$

On the other hand

$$
\left|m_{2^{j} B} u\right| \leq \sum_{k=1}^{j}\left|m_{2^{k} B} u-m_{2^{k-1} B} u\right|+\left|m_{B} u\right|
$$


and

$$
\text { (9) } \begin{aligned}
\left|m_{2^{k} B} u-m_{2^{k-1} B} u\right| & \leq 2^{n} \frac{1}{\left|2^{k} B\right|} \int_{2^{k} B}\left|u(y)-m_{2^{k} B} u\right| d y \\
& \leq 2^{n}\left\{\frac{1}{\left|2^{k} B\right|} \int_{2^{k} B}\left|u(y)-m_{2^{k} B} u\right|^{p} d y\right\}^{\frac{1}{p}} \\
& \leq 2^{n}\left|2^{k} B\right|^{\frac{1}{p}\left(\frac{\lambda}{n}-1\right)}\|u\|_{\mathcal{L}^{p, \lambda}\left(\mathbf{R}^{n}\right)}
\end{aligned}
$$

In the case $\lambda>n$, inequalities (7), (8) and (9) give

$$
\begin{aligned}
& m_{2^{j} B}|u|-\left|2^{j} B\right|^{\frac{1}{p}\left(\frac{\lambda}{n}-1\right)}\|u\|_{\mathcal{L}^{p, \lambda}\left(\mathbf{R}^{n}\right)} \\
& \leq \quad 2^{n}\left|2^{j} B\right|^{\frac{1}{p}\left(\frac{\lambda}{n}-1\right)}\left(\sum_{k=1}^{j} 2^{\frac{1}{p}(k-j)(\lambda-n)}\right)\|u\|_{\mathcal{L}^{p, \lambda}\left(\mathbf{R}^{n}\right)}+\left|m_{B} u\right| \\
& \leq C 2^{n}\left|2^{j} B\right|^{\frac{1}{p}\left(\frac{\lambda}{n}-1\right)}\|u\|_{\mathcal{L}^{p, \lambda}\left(\mathbf{R}^{n}\right)}+\left|m_{B} u\right|
\end{aligned}
$$

In the case $0 \leq \lambda \leq n$, we use (9) to get

$$
\left|m_{2^{k} B} u-m_{2^{k-1} B} u\right| \leq 2^{n}|B|^{\frac{1}{p}\left(\frac{\lambda}{n}-1\right)}\|u\|_{\mathcal{L}^{p, \lambda}\left(\mathbf{R}^{n}\right)}
$$

and by (7) and (8)

$$
m_{2^{j} B}|u|-\left|2^{j} B\right|^{\frac{1}{p}\left(\frac{\lambda}{n}-1\right)}\|u\|_{\mathcal{L}^{p, \lambda}\left(\mathbf{R}^{n}\right)} \leq j 2^{n}|B|^{\frac{1}{p}\left(\frac{\lambda}{n}-1\right)}\|u\|_{\mathcal{L}^{p, \lambda}\left(\mathbf{R}^{n}\right)}+\left|m_{B} u\right|
$$

The proof of Lemma 18 is complete.

Lemma 20. Let $s \in \mathbf{R}$ and $1 \leq p \leq+\infty$. Let $I^{s}=\mathcal{F}^{-1}\left\{|\cdot|^{-s} \mathcal{F}\right\}$ be the Riesz potential operator and $u \in \mathcal{Z}^{\prime}\left(\mathbf{R}^{n}\right)$ such that $\dot{\Delta}_{k} u \in L^{p}\left(\mathbf{R}^{n}\right)$ for any $k \in \mathbf{Z}$. Then

$$
\left\|I^{s} \dot{\Delta}_{k} u\right\|_{L^{p}\left(\mathbf{R}^{n}\right)} \leq C 2^{-k s}\|u\|_{L^{p}\left(\mathbf{R}^{n}\right)}
$$

holds and the constant $C$ is independent of $k$ and $u$.

Proof. Note that

$$
\dot{\Delta}_{k} u=\sum_{l=-1}^{1} \dot{\Delta}_{k+l} \dot{\Delta}_{k} u
$$

holds for any $k$. Thus, Young's inequality gives

$$
\left\|I^{s} \dot{\Delta}_{k} u\right\|_{L^{p}\left(\mathbf{R}^{n}\right)} \leq \sum_{l=-1}^{1}\left\|I^{s} \dot{\Delta}_{k+l} \dot{\Delta}_{k} u\right\|_{L^{p}\left(\mathbf{R}^{n}\right)}
$$




$$
\begin{aligned}
& \leq \sum_{l=-1}^{1}\left\|\mathcal{F}^{-1}\left(|\cdot|^{-s} \theta\left(2^{-k-l} \cdot\right)\right)\right\|_{L^{1}\left(\mathbf{R}^{n}\right)}\left\|\dot{\Delta}_{k} u\right\|_{L^{p}\left(\mathbf{R}^{n}\right)} \\
& \leq C\|u\|_{L^{p}\left(\mathbf{R}^{n}\right)} \sum_{l=-1}^{1}\left\|\mathcal{F}^{-1}\left(|\cdot|^{-s} \theta\left(2^{-k-l} \cdot\right)\right)\right\|_{L^{1}\left(\mathbf{R}^{n}\right)}
\end{aligned}
$$

Now

$$
\mathcal{F}^{-1}\left(|\cdot|^{-s} \theta\left(2^{-k-l} \cdot\right)\right)(x)=2^{-(k+l) s} 2^{(k+l) n} \mathcal{F}^{-1}\left(|\cdot|^{-s} \theta\right)\left(2^{k+l} x\right)
$$

yields

$$
\left\|\mathcal{F}^{-1}\left(|\cdot|^{-s} \theta\left(2^{-k-l} \cdot\right)\right)\right\|_{L^{1}\left(\mathbf{R}^{n}\right)}=2^{-(k+l) s}\left\|\mathcal{F}^{-1}\left(|\cdot|^{-s} \theta\right)\right\|_{L^{1}\left(\mathbf{R}^{n}\right)}
$$

and the proof is complete.

\section{Proof of Proposition 8}

(i) Let $f \in \dot{\mathcal{L}}^{2, \lambda, 0}\left(\mathbf{R}^{n}\right)$. Using Lemma 17 it suffices to show that for any ball $B$ centered at $x_{0} \in \mathbf{R}^{n}$ and with radius $2^{-J}, J \in \mathbf{Z}$, there exists a constant $C_{B} \in \mathbf{R}$ such that

$$
\sup _{B} \frac{1}{|B|^{\frac{\lambda}{n}}} \int_{B}\left|f-C_{B}\right|^{2} d x \leq C<+\infty .
$$

Let us take $C_{B}=\dot{S}_{J} f\left(x_{0}\right)$.

$$
\begin{aligned}
& \left(\frac{1}{|B|^{\frac{\lambda}{n}}} \int_{B}\left|f-\dot{S}_{J} f\left(x_{0}\right)\right|^{2} d x\right)^{\frac{1}{2}} \\
& \leq\left(\frac{1}{|B|^{\frac{\lambda}{n}}} \int_{B}\left|\dot{S}_{J} f(x)-\dot{S}_{J} f\left(x_{0}\right)\right|^{2} d x\right)^{\frac{1}{2}} \\
& +\left(\frac{1}{|B|^{\frac{\lambda}{n}}} \int_{B}\left|\sum_{j>J} \dot{\Delta}_{j} f(x)\right|^{2} d x\right)^{\frac{1}{2}}
\end{aligned}
$$

For any $x \in B$ we have

$$
\left|\dot{S}_{J} f(x)-\dot{S}_{J} f\left(x_{0}\right)\right| \leq 2^{-J} \sup _{x \in B}\left|\nabla \dot{S}_{J} f(x)\right|
$$


Lemma 13 yields in $Z^{\prime}\left(\mathbf{R}^{n}\right)$

$$
f=\sum_{j \in \mathbf{Z}} \dot{\Delta}_{j} f=\dot{S}_{J} f+\sum_{j>J} \dot{\Delta}_{j} f
$$

so $\dot{S}_{J} f=\sum_{j \leq J} \dot{\Delta}_{j} f$ in $Z^{\prime}\left(\mathbf{R}^{n}\right)$ and thus

$$
\nabla \dot{S}_{J} f(x)=\sum_{j \leq J} \nabla\left(\dot{\Delta}_{j} f\right)(x), x \in \mathbf{R}^{n}
$$

Let $\phi \in \mathcal{S}\left(\mathbf{R}^{n}\right)$ with $\mathcal{F} \phi=1$ on $\frac{1}{2} \leq|\xi| \leq 2$.

From $\mathcal{F} \dot{\Delta}_{j} u(\xi)=\mathcal{F}\left(\dot{\Delta}_{j} u\right)(\xi) \mathcal{F} \phi\left(2^{-j} \xi\right)$ we deduce

$$
\dot{\Delta}_{j} u(x)=2^{n j} \int_{\mathbf{R}^{n}} \dot{\Delta}_{j} u(y) \phi\left(2^{j}(x-y)\right) d y
$$

Thus for $j$ integer $\leq J$

$$
\nabla\left(\dot{\Delta}_{j} f\right)(x)=2^{(n+1) j} \int_{\mathbf{R}^{n}}\left(\dot{\Delta}_{j} f\right)(y)(\nabla \phi)\left(2^{j}(x-y)\right) d y
$$

We divide the integration domain $\mathbf{R}^{n}=E_{j} \cup \bigcup_{\nu=-j+1}^{\infty} F_{\nu}$ with

$E_{j}=\left\{y ;\left|y-x_{0}\right| \leq 2^{-j+1}\right\}$ and $F_{\nu}=\left\{y ; 2^{\nu} \leq\left|y-x_{0}\right| \leq 2^{\nu+1}\right\}, \nu \geq$ $-j+1$.

On $E_{j}$ we write

$$
\left|(\nabla \phi)\left(2^{j}(x-y)\right)\right| \leq\|\nabla \phi\|_{\infty} .
$$

Since $x \in B=B\left(x_{0}, 2^{-J}\right)$ and $j \leq J$, if $y \in F_{\nu}, \nu \geq-j+1 \geq-J+1$ then $\left|y-x_{0}\right| \sim|y-x|$ and $\left|2^{j}(x-y)\right|$ is large. Hence for each $N \in \mathbf{N}$, there exists $C_{N}>0$ such that

$$
\left|(\nabla \phi)\left(2^{j}(x-y)\right)\right| \leq C_{N} 2^{-(j+\nu) N}
$$

Therefore

$$
\begin{array}{r}
\sum_{j \leq J}\left|\nabla\left(\dot{\Delta}_{j} f\right)(x)\right| \leq C \sum_{j \leq J} 2^{j(n+1)}\left\{\int_{E_{j}}\left|\dot{\Delta}_{j} f(y)\right| d y+\right. \\
\left.\sum_{\nu=-j+1}^{\infty} 2^{-(j+\nu) N} \int_{F_{\nu}}\left|\dot{\Delta}_{j} f(y)\right| d y\right\}
\end{array}
$$


Now hypothesis $f \in \dot{\mathcal{L}}^{2, \lambda, 0}\left(\mathbf{R}^{n}\right)$ yields in particular a constant $C>0$ such that for any ball $\widetilde{B}$ of $\mathbf{R}^{n}$ of radius $2^{-\widetilde{J}}, \widetilde{J} \in \mathbf{Z}$, we get for any $k \geq \widetilde{J}$

$$
\int_{\widetilde{B}}\left|\dot{\Delta}_{k} f(y)\right| d y \leq C|\widetilde{B}|^{\frac{1}{2}+\frac{\lambda}{2 n}}\|f\|_{\dot{\mathcal{L}}^{2, \lambda, 0}\left(\mathbf{R}^{n}\right)}
$$

For $\int_{E_{j}}\left|\dot{\Delta}_{j} f(y)\right| d y$ the condition $k \geq \widetilde{J}$ becomes $j \geq j-1$; and for $\int_{F_{\nu}}\left|\dot{\Delta}_{j} f(y)\right| d y$ the condition $k \geq \widetilde{J}$ becomes $j \geq-\nu-1$. So we can apply the above inequality to these two terms, and we deduce for $N$ large and $x \in B($ since $0 \leq \lambda<n+2)$

$$
\begin{aligned}
\sum_{j \leq J}\left|\nabla\left(\dot{\Delta}_{j} f\right)(x)\right| \leq & C\|f\|_{\dot{\mathcal{L}}^{2, \lambda, 0}{ }_{\left(\mathbf{R}^{n}\right)}}\left\{\sum_{j \leq J} 2^{j \frac{n+2-\lambda}{2}}\right. \\
& \left.+\sum_{j \leq J} 2^{j(n+1)} \sum_{\nu=-j+1}^{\infty} 2^{-(j+\nu) N} 2^{\nu n\left(\frac{1}{2}+\frac{\lambda}{2 n}\right)}\right\} \\
\leq & C\|f\|_{\dot{\mathcal{L}}^{2, \lambda, 0}{ }_{\left(\mathbf{R}^{n}\right)}} \sum_{j \leq J} 2^{j \frac{n+2-\lambda}{2}} \\
\leq & C\|f\|_{\dot{\mathcal{L}}^{2, \lambda, 0}\left(\mathbf{R}^{n}\right)} 2^{\frac{n+2-\lambda}{2} J}
\end{aligned}
$$

Therefore

$$
\left(\frac{1}{|B|^{\frac{\lambda}{n}}} \int_{B}\left|\dot{S}_{J} f(x)-\dot{S}_{J} f\left(x_{0}\right)\right|^{2} d x\right)^{\frac{1}{2}} \leq C\|f\|_{\dot{\mathcal{L}}^{2, \lambda, 0}\left(\mathbf{R}^{n}\right)}
$$

Now we show the following inequality

$$
\frac{1}{|B|^{\frac{\lambda}{n}}} \int_{B}\left|\sum_{j>J} \dot{\Delta}_{j} f(x)\right|^{2} d x \leq C\|f\|_{\dot{\mathcal{L}}^{2, \lambda, 0}\left(\mathbf{R}^{n}\right)}^{2}
$$

For this, let $\theta \in C_{0}^{\infty}\left(\mathbf{R}^{n}\right)$ be a positive function equal 0 on $|\xi| \geq \frac{1}{2}$ such that $\theta(-\xi)=\theta(\xi)$ and $\int \theta(\xi) d \xi=2(2 \pi)^{n}$. Then $\mathcal{F}^{-1} \theta(x) \geq 1$ for $|x| \leq 1$. [For this let us take $n=1: \mathcal{F}^{-1} \theta(x)=\frac{1}{2 \pi} \int e^{i x \xi} \theta(\xi) d \xi=\frac{1}{\pi} \int_{0}^{1 / 2} e^{i x \xi} \theta(\xi) d \xi$, so $\mathcal{F}^{-1} \theta(0)=\frac{1}{\pi} \int_{0}^{1 / 2} \theta(\xi) d \xi=2$ and $\overline{\mathcal{F}^{-1} \theta(x)}=\mathcal{F}^{-1} \theta(x)$. We deduce for $|x| \leq 1$,

$$
\left|\mathcal{F}^{-1} \theta(x)-\mathcal{F}^{-1} \theta(0)\right|=\frac{1}{\pi}\left|\int_{0}^{1 / 2}\left(e^{i x \xi}-1\right) \theta(\xi) d \xi\right|
$$




$$
\begin{aligned}
& =\frac{|x|}{\pi}\left|\int_{0}^{1 / 2} \xi e^{i c \xi} \theta(\xi) d \xi\right| \\
& \leq \frac{1}{2 \pi} \int_{0}^{1 / 2}|\theta(\xi)| d \xi=1 .
\end{aligned}
$$

Thus $\left|\mathcal{F}^{-1} \theta(x)-2\right| \leq 1$ for all $\left.|x| \leq 1\right]$.

We set now

$$
\psi(x)=2^{J \frac{\lambda}{2}} \mathcal{F}^{-1} \theta\left(2^{J}\left(x-x_{0}\right)\right)
$$

We have supp $\mathcal{F} \psi \subset\left\{|\xi| \leq \frac{1}{2} 2^{J}\right\}$ and $\psi(x)^{2} \geq \frac{C}{|B|^{\frac{\lambda}{n}}}$ for $x \in B$. Thus

$$
\begin{aligned}
\frac{1}{|B|^{\frac{\lambda}{n}}} \int_{B}\left|\sum_{j>J} \dot{\Delta}_{j} f(x)\right|^{2} d x & \leq C \int_{B}\left|\sum_{j>J} \psi(x) \dot{\Delta}_{j} f(x)\right|^{2} d x \\
& \leq C \int_{\mathbf{R}^{n}}\left|\sum_{j>J} \mathcal{F}\left(\psi \dot{\Delta}_{j} f\right)(\xi)\right|^{2} d \xi
\end{aligned}
$$

For $j>J$

$$
\operatorname{supp} \mathcal{F}\left(\psi \dot{\Delta}_{j} f\right) \subset\left\{\frac{1}{2}\left(2^{j}-2^{J}\right) \leq|\xi| \leq \frac{5}{2} 2^{j}\right\}
$$

implies for each fixed $\xi \in \mathbf{R}^{n}$ satisfying $|\xi|<\frac{1}{2}\left(2^{j}-2^{J}\right), \mathcal{F}\left(\psi \dot{\Delta}_{j} f\right)(\xi)=0$. Then, $\sum_{j>J} \mathcal{F}\left(\psi \dot{\Delta}_{j} f\right)(\xi)=\sum_{j=J+1}^{N} \mathcal{F}\left(\psi \dot{\Delta}_{j} f\right)(\xi)$ where $N=N(\xi, J)$ is any integer $>\log _{2}\left(2|\xi|+2^{J}\right)$. Consequently,

$$
\begin{aligned}
\left|\sum_{j>J} \mathcal{F}\left(\psi \dot{\Delta}_{j} f\right)(\xi)\right|^{2} & \leq\left\{\sum_{j=J+1}^{N} \mid \mathcal{F}\left(\psi \dot{\Delta}_{j} f\right)(\xi)\right\}^{2} \\
= & \sum_{j=J+1}^{N}\left|\mathcal{F}\left(\psi \dot{\Delta}_{j} f\right)(\xi)\right|^{2} \\
& +2 \sum_{j, k=J+1}^{N}\left|\mathcal{F}\left(\psi \dot{\Delta}_{j} f\right)(\xi)\right|\left|\mathcal{F}\left(\psi \dot{\Delta}_{k} f\right)(\xi)\right| \\
& =\sum_{j=J+1}^{N}\left|\mathcal{F}\left(\psi \dot{\Delta}_{j} f\right)(\xi)\right|^{2}
\end{aligned}
$$




$$
\begin{aligned}
& +2 \sum_{j=J+1}^{N}\left|\mathcal{F}\left(\psi \dot{\Delta}_{j} f\right)(\xi)\right|\left|\mathcal{F}\left(\psi \dot{\Delta}_{j+1} f\right)(\xi)\right| \\
& +2 \sum_{j=J+1}^{N}\left|\mathcal{F}\left(\psi \dot{\Delta}_{j} f\right)(\xi)\right|\left|\mathcal{F}\left(\psi \dot{\Delta}_{j+2} f\right)(\xi)\right| \\
& +2 \sum_{j=J+1}^{N}\left|\mathcal{F}\left(\psi \dot{\Delta}_{j} f\right)(\xi)\right|\left|\mathcal{F}\left(\psi \dot{\Delta}_{j+3} f\right)(\xi)\right| \\
& \leq \quad 7 \sum_{j=J+1}^{N}\left|\mathcal{F}\left(\psi \dot{\Delta}_{j} f\right)(\xi)\right|^{2} \\
& =7 \sum_{j>J}\left|\mathcal{F}\left(\psi \dot{\Delta}_{j} f\right)(\xi)\right|^{2}
\end{aligned}
$$

With (14), (15) and Plancherel theorem there exists a constant $C$ independent of $J, B$ and $\xi$ such that

$$
\begin{aligned}
\frac{1}{|B|^{\frac{\lambda}{n}}} \int_{B}\left|\sum_{j>J} \dot{\Delta}_{j} f(x)\right|^{2} d x & \leq C \int_{\mathbf{R}^{n}} \sum_{j>J}\left|\mathcal{F}\left(\psi \dot{\Delta}_{j} f\right)(\xi)\right|^{2} d \xi \\
& \leq C \sum_{j>J} \int_{\mathbf{R}^{n}}\left|\psi(x) \dot{\Delta}_{j} f(x)\right|^{2} d x
\end{aligned}
$$

We decompose $\mathbf{R}^{n}$ into disjoint cubes $Q_{\nu}=\left\{x \in \mathbf{R}^{n} /\left\|x-x_{0}-2^{-J} \nu\right\|_{\infty} \leq 2^{-J-1}\right\}, \nu \in \mathbf{Z}^{n}$, and we set $a_{\nu}=\sup _{\|x-\nu\|_{\infty} \leq \frac{1}{2}}\left|\mathcal{F}^{-1} \theta(x)\right|^{2}$ which is a rapidly decreasing sequence.

Thus,

$$
\begin{aligned}
\frac{1}{|B|^{\frac{\lambda}{n}}} \int_{B}\left|\sum_{j>J} \dot{\Delta}_{j} f(x)\right|^{2} d x & \leq C \sum_{\nu} \sum_{j \geq J} \int_{Q_{\nu}}\left|\psi(x) \dot{\Delta}_{j} f(x)\right|^{2} d x \\
& \leq C \sum_{\nu} a_{\nu} \frac{1}{\left|Q_{\nu}\right|^{\frac{\lambda}{n}}} \int_{Q_{\nu}} \sum_{j \geq J}\left|\dot{\Delta}_{j} f(x)\right|^{2} d x \\
& \leq C\|f\|_{\dot{\mathcal{L}}^{2, \lambda, 0}\left(\mathbf{R}^{n}\right)}^{2} \sum_{\nu} a_{\nu}
\end{aligned}
$$

Inequality (13) is proved. Finally combining (10), (12) and (13) we obtain

$$
\left(\frac{1}{|B|^{\frac{\lambda}{n}}} \int_{B}\left|f-\dot{S}_{J} f\left(x_{0}\right)\right|^{2} d x\right)^{\frac{1}{2}} \leq C\|f\|_{\dot{\mathcal{L}}^{2, \lambda, 0}}\left(\mathbf{R}^{n}\right)
$$


The injection $\dot{\mathcal{L}}^{2, \lambda, 0}\left(\mathbf{R}^{n}\right) \hookrightarrow \dot{\mathcal{L}}^{2, \lambda}\left(\mathbf{R}^{n}\right)$ is proved for $0 \leq \lambda<n+2$.

Conversely let $f \in \dot{\mathcal{L}}^{2, \lambda}\left(\mathbf{R}^{n}\right)$.

We decompose $f=f_{1}+f_{2}+f_{3}$ with $f_{1}=m_{2 B} f, f_{2}=\left(f-m_{2 B} f\right) \chi_{2 B}$ and $f_{3}=\left(f-m_{2 B} f\right) \chi_{\mathbf{R}^{n} \backslash 2 B}$ where $B$ is a ball of $\mathbf{R}^{n}$ of radius $2^{-J}, J \in \mathbf{Z}$, and $\chi_{\Omega}$ is the characteristic function of the set $\Omega$.

The function $f_{1}$ is constant, $\dot{\Delta}_{k} f_{1}=0$ for any $k$.

For $f_{2}$ we have

$$
\begin{aligned}
\left\|f_{2}\right\|_{L^{2}\left(\mathbf{R}^{n}\right)} & =\left(\int_{2 B}\left|f-m_{2 B} f\right|^{2} d x\right)^{1 / 2} \\
& \leq C|B|^{\frac{\lambda}{2 n}}\|f\|_{\dot{\mathcal{L}}^{2, \lambda}\left(\mathbf{R}^{n}\right)}
\end{aligned}
$$

Now $\dot{F}_{2,2}^{0}\left(\mathbf{R}^{n}\right) \equiv L^{2}\left(\mathbf{R}^{n}\right)$ yields

$$
\begin{aligned}
\left(\sum_{j \geq J}\left\|\dot{\Delta}_{j} f_{2}\right\|_{L^{2}(B)}^{2}\right)^{\frac{1}{2}} & \leq\left(\sum_{j \in \mathbf{Z}}\left\|\dot{\Delta}_{j} f_{2}\right\|_{L^{2}\left(\mathbf{R}^{n}\right)}^{2}\right)^{\frac{1}{2}} \\
& \leq C\left\|f_{2}\right\|_{L^{2}\left(\mathbf{R}^{n}\right)} \\
& \leq C|B|^{\frac{\lambda}{2 n}}\|f\|_{\dot{\mathcal{L}}^{2, \lambda}\left(\mathbf{R}^{n}\right)}
\end{aligned}
$$

For $f_{3}$ we will show that for any $x \in B, j \geq J$ and $0 \leq \lambda<n+2$

$$
\left|\dot{\Delta}_{j} f_{3}(x)\right| \leq C 2^{J-j}|B|^{\frac{1}{2}\left(\frac{\lambda}{n}-1\right)}\|f\|_{\dot{\mathcal{L}}^{2, \lambda}\left(\mathbf{R}^{n}\right)}
$$

holds.

From inequality (17) we deduce

$$
\sum_{j \geq J}\left\|\dot{\Delta}_{j} f_{3}\right\|_{L^{2}(B)}^{2} \leq C|B|^{\frac{\lambda}{n}}\|f\|_{\dot{\mathcal{L}}^{2, \lambda}\left(\mathbf{R}^{n}\right)}^{2}
$$

and the proof of the injection $\dot{\mathcal{L}}^{2, \lambda}\left(\mathbf{R}^{n}\right) \hookrightarrow \dot{\mathcal{L}}^{2, \lambda, 0}\left(\mathbf{R}^{n}\right)$ for $0 \leq \lambda<n+2$ will be complete.

Proof of (17). For $x \in B$

$$
\dot{\Delta}_{j} f_{3}(x)=2^{j n} \int_{\mathbf{R}^{n} \backslash 2 B} f_{3}(y) \mathcal{F}^{-1} \theta\left(2^{j}(x-y)\right) d y
$$


Now $\mathcal{F}^{-1} \theta \in \mathcal{S}\left(\mathbf{R}^{n}\right)$ yields a constant $C>0$ such that

$$
\left|\mathcal{F}^{-1} \theta(x)\right| \leq C|x|^{-n-1} \text { for }|x| \geq 1 .
$$

If $x \in B, y \in \mathbf{R}^{n} \backslash 2 B$ and $j \geq J$, then $2^{j}|x-y| \geq 1$ and $\left|x_{0}-y\right| \leq$ $2|x-y|$. So

$$
\begin{aligned}
\left|\dot{\Delta}_{j} f_{3}(x)\right| & \leq C 2^{-j} \int_{\mathbf{R}^{n} \backslash 2 B}\left|f-m_{2 B} f\right|(y)\left|x_{0}-y\right|^{-n-1} d y \\
& \leq C 2^{-j} \sum_{l=1}^{+\infty} \int_{2^{l+1} B \backslash 2^{l} B}\left|f-m_{2 B} f\right|(y)\left|x_{0}-y\right|^{-n-1} d y \\
& \leq C 2^{J-j} \sum_{l=1}^{+\infty} 2^{-l} m_{2^{l+1} B}\left(\left|f-m_{2 B} f\right|\right)
\end{aligned}
$$

Using Lemma 18 we obtain

$$
\begin{aligned}
\left|\dot{\Delta}_{j} f_{3}(x)\right| \leq C 2^{J-j} & \sum_{l=1}^{+\infty} 2^{-l}\left\{\left|m_{2 B}\left(f-m_{2 B} f\right)\right|\right. \\
& \left.+C(l+1)\left|2^{\alpha l} B\right|^{\frac{1}{2}\left(\frac{\lambda}{n}-1\right)}\left\|f-m_{2 B} f\right\|_{\dot{\mathcal{L}}^{2, \lambda}\left(\mathbf{R}^{n}\right)}\right\} \\
\leq C 2^{J-j} & \sum_{l=1}^{+\infty} 2^{-l\left(1-\frac{\alpha}{2} n\left(\frac{\lambda}{n}-1\right)\right)}(l+1)\|f\|_{\dot{\mathcal{L}}^{2, \lambda}\left(\mathbf{R}^{n}\right)}
\end{aligned}
$$

where $\alpha=\left\{\begin{array}{lll}0 & \text { if } \quad 0 \leq \lambda \leq n \\ 1 & \text { if } \quad \lambda>n\end{array}\right.$. The series converges when $\lambda<n+2$. Inequality (17) and then the first part of Proposition 8 are proved.

(ii) Let $f \in \mathcal{L}^{2, \lambda, 0}\left(\mathbf{R}^{n}\right)$. We show that for any ball $B$ centered at $x_{0} \in \mathbf{R}^{n}$ and with radius $2^{-J}, J \in \mathbf{Z}$, there exists a constant $C_{B} \in \mathbf{R}$ such that

$$
\sup _{B, J \geq 0} \frac{1}{|B|^{\frac{\lambda}{n}}} \int_{B}\left|f-C_{B}\right|^{2} d x+\sup _{B, J=0} \frac{1}{|B|} \int_{B}|f|^{2} d x=C<+\infty .
$$

To estimate $\sup _{B, J \geq 0} \frac{1}{|B|^{\frac{\lambda}{n}}} \int_{B}\left|f-C_{B}\right|^{2} d x$, we write

$$
f=\sum_{j \geq 0} \Delta_{j} f=\Delta_{0} f+\widetilde{S}_{J^{+}} f+\sum_{j>J^{+}} \Delta_{j} f, \text { here } J^{+}=J \geq 0 .
$$


where $\widetilde{S}_{J^{+}} f=\sum_{j=1}^{J^{+}} \Delta_{j} f$. Set $C_{B}=\widetilde{S}_{J^{+}} f\left(x_{0}\right)$. Thus

$$
\begin{aligned}
& \left(\frac{1}{|B|^{\frac{\lambda}{n}}} \int_{B}\left|f-\widetilde{S}_{J^{+}} f\left(x_{0}\right)\right|^{2} d x\right)^{\frac{1}{2}} \\
& \leq\left(\frac{1}{|B|^{\frac{\lambda}{n}}} \int_{B}\left|\Delta_{0} f\right|^{2} d x\right)^{\frac{1}{2}}+\left(\frac{1}{|B|^{\frac{\lambda}{n}}} \int_{B}\left|\widetilde{S}_{J^{+}} f(x)-\widetilde{S}_{J^{+}} f\left(x_{0}\right)\right|^{2} d x\right)^{\frac{1}{2}} \\
& \quad+\left(\frac{1}{|B|^{\frac{\lambda}{n}}} \int_{B}\left|\sum_{j>J^{+}} \Delta_{j} f(x)\right|^{2} d x\right)^{\frac{1}{2}}
\end{aligned}
$$

The first term of the right hand side is bounded from above by $\|f\|_{\mathcal{L}^{2, \lambda, 0}\left(\mathbf{R}^{n}\right)}$. For the two other terms we argue in the same way as for inequalities (12) and (13).

To estimate the remainder term $\sup _{B, J=0} \frac{1}{|B|} \int_{B}|f|^{2} d x$ we write

$$
\left(\int_{B}|f|^{2} d x\right)^{1 / 2} \leq C\left\{\left\|\Delta_{0} f\right\|_{L^{2}(B)}+\left(\int_{B}\left|\sum_{j \geq 1} \Delta_{j} f(x)\right|^{2} d x\right)^{1 / 2}\right\}
$$

In the same way as for inequality (13) we deduce

$$
\left(\int_{B}|f|^{2} d x\right)^{1 / 2} \leq C\|f\|_{\mathcal{L}^{2, \lambda, 0}\left(\mathbf{R}^{n}\right)} .
$$

Conversely, let $f \in l^{2, \lambda}$. We use the same decomposition $f=f_{1}+f_{2}+f_{3}$ as in the first part.

For $f_{1}$, if $J \geq 1$ then $\Delta_{j} f_{1}=0$ for $j \geq 1$, and $\left(\frac{1}{|B|^{\frac{\lambda}{n}}} \sum_{j \geq J^{+}}\left\|\Delta_{j} f_{1}\right\|_{L^{2}(B)}^{2}\right)^{1 / 2}$ $=0 \leq\|f\|_{l^{2, \lambda}}$.

In the case $J \leq 0$, we remark that $\Delta_{0} f_{1}=f_{1}$ and $J^{+}=0$, so

$$
\begin{aligned}
\left(\frac{1}{|B|^{\frac{\lambda}{n}}} \sum_{j \geq 0}\left\|\Delta_{j} f_{1}\right\|_{L^{2}(B)}^{2}\right)^{1 / 2} & =\left(\frac{1}{|B|^{\frac{\lambda}{n}}} \int_{B}\left|\Delta_{0} f_{1}\right|^{2} d x\right)^{1 / 2} \\
& =\frac{1}{|B|^{\frac{\lambda}{2 n}-\frac{1}{2}}}\left|m_{2 B} f\right|
\end{aligned}
$$




$$
\leq C\left(\frac{1}{|2 B|^{\frac{\lambda}{n}}} \int_{2 B}|f|^{2} d x\right)^{1 / 2}
$$

this last term is bounded from above by $C\|f\|_{l^{2, \lambda}}$ since (3) is valid with $P_{B}=0$ when $\delta=2^{-J} \geq 1$.

For $f_{2}$ and $f_{3}$ we argue in the same way as for (16) and (18) and we use the injection $l^{2, \lambda} \hookrightarrow \mathcal{L}^{2, \lambda}\left(\mathbf{R}^{n}\right)$.

In order to compare between the spaces $\dot{\mathcal{L}}^{p, \lambda, 0}\left(\mathbf{R}^{n}\right)$ and $\dot{\mathcal{L}}^{p, \lambda}\left(\mathbf{R}^{n}\right)$ for $p \geq 1$, we consider two cases $p \geq 2$ and $1 \leq p \leq 2$ :

Proposition 21. Let $p \geq 2$, and $0 \leq \lambda<n+p$. The Campanato space $\dot{\mathcal{L}}^{p, \lambda}\left(\mathbf{R}^{n}\right)$ is continuously embedded in the space $\dot{\mathcal{L}}^{p, \lambda, 0}\left(\mathbf{R}^{n}\right)$.

Proof. The proof is similar to the one of Proposition 8. Let $f \in$ $\dot{\mathcal{L}}^{p, \lambda}\left(\mathbf{R}^{n}\right)$. We decompose $f=f_{1}+f_{2}+f_{3}$ with $f_{1}=m_{2 B} f, f_{2}=$ $\left(f-m_{2 B} f\right) \chi_{2 B}$ and $f_{3}=\left(f-m_{2 B} f\right) \chi_{\mathbf{R}^{n} \backslash 2 B}$ where $B$ is a ball of $\mathbf{R}^{n}$ of radius $2^{-J}, J \in \mathbf{Z}$.

The function $f_{1}$ is constant, $\dot{\Delta}_{k} f_{1}=0$ for any $k$. For the function $f_{2}$ we have $\left\|f_{2}\right\|_{L^{p}\left(\mathbf{R}^{n}\right)} \leq|2 B|^{\frac{\lambda}{n p}}\|f\|_{\dot{\mathcal{L}}^{p, \lambda}\left(\mathbf{R}^{n}\right)}$ then $f_{2} \in L^{p}\left(\mathbf{R}^{n}\right)$ and since $p \geq 2$ we obtain

$$
\begin{aligned}
\left(\sum_{j \geq J}\left\|\dot{\Delta}_{j} f_{2}\right\|_{L^{p}(B)}^{p}\right)^{\frac{1}{p}} & \leq\left(\sum_{j \in \mathbf{Z}}\left\|\dot{\Delta}_{j} f_{2}\right\|_{L^{p}\left(\mathbf{R}^{n}\right)}^{p}\right)^{\frac{1}{p}} \\
& =\left\|f_{2}\right\|_{\dot{B}_{p, p}^{0}\left(\mathbf{R}^{n}\right)} \\
& \leq C\left\|f_{2}\right\|_{L^{p}\left(\mathbf{R}^{n}\right)} \\
& \leq C|B|^{\frac{\lambda}{n p}}\|f\|_{\dot{\mathcal{L}}^{p, \lambda}\left(\mathbf{R}^{n}\right)}
\end{aligned}
$$

For $f_{3}$ we show in the same way as for inequality (17) that for any $x \in B$, $j \geq J$ and $\lambda<n+p$

$$
\left|\dot{\Delta}_{j} f_{3}(x)\right| \leq C 2^{J-j}|B|^{\frac{1}{p}\left(\frac{\lambda}{n}-1\right)}\|f\|_{\dot{\mathcal{L}}^{p, \lambda}\left(\mathbf{R}^{n}\right)}
$$

holds. So

$$
\sum_{j \geq J}\left\|\dot{\Delta}_{j} f_{3}\right\|_{L^{p}(B)}^{p} \leq C|B|^{\frac{\lambda}{n}}\|f\|_{\dot{\mathcal{L}}^{p, \lambda}\left(\mathbf{R}^{n}\right)}^{p}
$$

which complete the proof. 
Proposition 22. Let $1 \leq p \leq 2$ and $n-\frac{n}{2} p \leq \lambda<n+p$. The space $\dot{\mathcal{L}}^{p, \lambda, 0}\left(\mathbf{R}^{n}\right) \cap \dot{F}_{2, q}^{\frac{n}{2}+\frac{\lambda-n}{p}}\left(\mathbf{R}^{n}\right)$ is continuously embedded in Campanato space $\dot{\mathcal{L}}^{p, \lambda}\left(\mathbf{R}^{n}\right)$ for $1 \leq q \leq 2$.

Remark 23. If $n \leq \lambda<n+p$ and $1 \leq p \leq 2$ then

$$
\dot{\mathcal{L}}^{p, \lambda, 0}\left(\mathbf{R}^{n}\right) \cap \dot{F}_{2, q}^{\frac{n}{2}+\frac{\lambda-n}{p}}\left(\mathbf{R}^{n}\right) \hookrightarrow \dot{\mathcal{L}}^{p, \lambda, 0}\left(\mathbf{R}^{n}\right) \cap \dot{\mathcal{L}}^{2, \lambda}\left(\mathbf{R}^{n}\right)
$$

for $1 \leq q \leq 2$.

For this, Lemma 12 yields $\dot{F}_{2, q}^{\frac{n}{2}+\frac{\lambda-n}{p}}\left(\mathbf{R}^{n}\right) \hookrightarrow \dot{\mathcal{L}}^{2, \lambda, \frac{\lambda-n}{p}-\frac{\lambda-n}{2}}\left(\mathbf{R}^{n}\right)$, and $\lambda \geq n$ implies that $\dot{\mathcal{L}}^{2, \lambda, \frac{\lambda-n}{p}-\frac{\lambda-n}{2}}\left(\mathbf{R}^{n}\right) \hookrightarrow \dot{\mathcal{L}}^{2, \lambda, 0}\left(\mathbf{R}^{n}\right)=\dot{\mathcal{L}}^{2, \lambda}\left(\mathbf{R}^{n}\right)$.

Proof. It suffices to prove the proposition for $q=2$.

Let $f \in \dot{\mathcal{L}}^{p, \lambda, 0}\left(\mathbf{R}^{n}\right) \cap \dot{F}_{2,2}^{\frac{n}{2}+\frac{\lambda-n}{p}}\left(\mathbf{R}^{n}\right)$. Using Lemma 17 it suffices to show that for any ball $B$ centered at $x_{0} \in \mathbf{R}^{n}$ and with radius $2^{-J}, J \in \mathbf{Z}$, there exists a constant $C_{B} \in \mathbf{R}$ such that

$$
\sup _{B} \frac{1}{|B|^{\frac{\lambda}{n}}} \int_{B}\left|f-C_{B}\right|^{p} d x \leq C<+\infty
$$

Set $C_{B}=\dot{S}_{J} f\left(x_{0}\right)$. As in the proof of Proposition 8 we write

$$
\begin{aligned}
\left(\frac{1}{|B|^{\frac{\lambda}{n}}} \int_{B}\left|f-\dot{S}_{J} f\left(x_{0}\right)\right|^{p} d x\right)^{\frac{1}{p}} \leq & \left(\frac{1}{|B|^{\frac{\lambda}{n}}} \int_{B}\left|\dot{S}_{J} f(x)-\dot{S}_{J} f\left(x_{0}\right)\right|^{p} d x\right)^{\frac{1}{p}} \\
& +\left(\frac{1}{|B|^{\frac{\lambda}{n}}} \int_{B}\left|\sum_{j>J} \dot{\Delta}_{j} f(x)\right|^{p} d x\right)^{\frac{1}{p}}
\end{aligned}
$$

In the same way as for inequality (12) we obtain

$$
\left(\frac{1}{|B|^{\frac{\lambda}{n}}} \int_{B}\left|\dot{S}_{J} f(x)-\dot{S}_{J} f\left(x_{0}\right)\right|^{p} d x\right)^{\frac{1}{p}} \leq C\|f\|_{\dot{\mathcal{L}}^{p, \lambda, 0}\left(\mathbf{R}^{n}\right)}
$$

We will show

$$
\left\{\frac{1}{|B|^{\frac{\lambda}{n}}} \int_{B}\left|\sum_{j>J} \dot{\Delta}_{j} f(x)\right|^{p} d x\right\}^{1 / p} \leq C\|f\|_{\dot{F}_{2,2}^{\frac{n}{2}+\frac{\lambda-n}{p}}\left(\mathbf{R}^{n}\right)}
$$


For this, $p \leq 2$ and Plancherel theorem yield

$$
\begin{aligned}
& \left\{\frac{1}{|B|^{\frac{\lambda}{n}}} \int_{B}\left|\sum_{j>J} \dot{\Delta}_{j} f(x)\right|^{p} d x\right\}^{1 / p} \\
& \leq \frac{1}{|B|^{\frac{\lambda}{n p}}}\left\{\int_{B}\left|\sum_{j \geq J} \dot{\Delta}_{j} f(x)\right|^{2} d x\right\}^{1 / 2}|B|^{\frac{1}{p}-\frac{1}{2}} \\
& \leq C|B|^{-\frac{\lambda}{n p}+\frac{1}{p}-\frac{1}{2}}\left\{\int_{\mathbf{R}^{n}}\left|\sum_{j \geq J} \mathcal{F} \dot{\Delta}_{j} f(\xi)\right|^{2} d \xi\right\}^{1 / 2}
\end{aligned}
$$

We argue as for (15), and next we use the assumption $n-\frac{n}{2} p \leq \lambda$ to obtain

$$
\begin{aligned}
& \left\{\frac{1}{|B|^{\frac{\lambda}{n}}} \int_{B}\left|\sum_{j>J} \dot{\Delta}_{j} f(x)\right|^{p} d x\right\}^{1 / p} \\
& \leq C|B|^{-\frac{\lambda}{n p}+\frac{1}{p}-\frac{1}{2}}\left\{\int_{\mathbf{R}^{n}} \sum_{j>J}\left|\dot{\Delta}_{j} f(x)\right|^{2} d x\right\}^{1 / 2} \\
& \leq C\left\{\int_{\mathbf{R}^{n}} \sum_{j>J} 2^{2 J\left(\frac{n}{2}+\frac{\lambda-n}{p}\right)}\left|\dot{\Delta}_{j} f(x)\right|^{2} d x\right\}^{1 / 2} \\
& \leq C\left\{\int_{\mathbf{R}^{n}} \sum_{j>J} 2^{2 j\left(\frac{n}{2}+\frac{\lambda-n}{p}\right)}\left|\dot{\Delta}_{j} f(x)\right|^{2} d x\right\}^{1 / 2} \\
& \leq C\left\{\int_{\mathbf{R}^{n}} \sum_{j \in \mathbf{Z}} 2^{2 j\left(\frac{n}{2}+\frac{\lambda-n}{p}\right)}\left|\dot{\Delta}_{j} f(x)\right|^{2} d x\right\}^{1 / 2} \\
& =C\|f\|_{\dot{F}_{2,2}^{\frac{n}{2}+\frac{\lambda-n}{p}}\left(\mathbf{R}^{n}\right)}
\end{aligned}
$$

Finally, with (20) and (21) we deduce

$$
\left(\frac{1}{|B|^{\frac{\lambda}{n}}} \int_{B}\left|f-\dot{S}_{J} f\left(x_{0}\right)\right|^{p} d x\right)^{\frac{1}{p}} \leq C\left(\|f\|_{\dot{\mathcal{L}}^{p, \lambda, 0}\left(\mathbf{R}^{n}\right)}+\|f\|_{\dot{F}_{2,2}^{\frac{n}{2}+\frac{\lambda-n}{p}}\left(\mathbf{R}^{n}\right)}\right)
$$

which complete the proof. 
Proof of Corollary 9. We recall that for $m=0, \dot{\mathcal{L}}^{2, \lambda, 0}\left(\mathbf{R}^{n}\right)=\dot{\mathcal{L}}^{2, \lambda}\left(\mathbf{R}^{n}\right)=$ $\dot{\mathcal{H}}^{2, \lambda, 0}$.

(i) If $u \in \dot{\mathcal{L}}^{2, \lambda, m}\left(\mathbf{R}^{n}\right)$ then $D^{\alpha} u \in \dot{\mathcal{L}}^{2, \lambda, m-|\alpha|}\left(\mathbf{R}^{n}\right) \hookrightarrow \dot{\mathcal{L}}^{2, \lambda, 0}\left(\mathbf{R}^{n}\right)=$ $\dot{\mathcal{L}}^{2, \lambda}\left(\mathbf{R}^{n}\right),|\alpha| \leq m$.

(ii) Let $u \in \dot{\mathcal{H}}^{2, \lambda, m} \cap \dot{H}^{m+\lambda / 2}\left(\mathbf{R}^{n}\right)$. Inequality (6) yields

$$
\begin{aligned}
& \frac{1}{|B|^{\frac{\lambda}{n}}} \sum_{l \geq J} 2^{2 l m}\left\|\dot{\Delta}_{l} u\right\|_{L^{2}(B)}^{2} \leq \frac{C}{|B|^{\frac{\lambda}{n}}} \sum_{l \geq J} 2^{2 l m} \sum_{j \sim l}\left\|\dot{\Delta}_{j} \dot{\Delta}_{l} u\right\|_{L^{2}(B)}^{2} \\
& \leq \frac{C}{|B|^{\frac{\lambda}{n}}} \sum_{l \geq J} 2^{2 l m} 2^{-2 l m} \sup _{|\alpha| \leq m}\left\|D^{\alpha} \dot{\Delta}_{l} u\right\|_{L^{2}(3 B)}^{2} \\
& +\frac{C}{|B|^{\frac{\lambda}{n}}} \sum_{l \geq J} 2^{2 l m} 2^{-2(l-J) M}\left\|\dot{\Delta}_{l} u\right\|_{L^{2}\left(\mathbf{R}^{n}\right)}^{2} \\
& \leq C \sup _{|\alpha| \leq m}\left\|D^{\alpha} u\right\|_{\dot{\mathcal{L}}^{2, \lambda, 0}}^{2}\left(\mathbf{R}^{n}\right) \\
& +C \sum_{l \geq J} 2^{2 l\left(m+\frac{\lambda}{2}\right)}\left\|\dot{\Delta}_{l} u\right\|_{L^{2}\left(\mathbf{R}^{n}\right)}^{2} \\
& \leq C\|u\|_{\dot{\mathcal{H}}^{2, \lambda, m}}^{2}+C\|u\|_{\dot{H}^{m+\frac{\lambda}{2}}\left(\mathbf{R}^{n}\right)}^{2} .
\end{aligned}
$$

\section{Proof of Theorem 10}

The first part of Theorem 10 follows from these following lemmas.

Lemma 24. There exists a constant $C>0$ such that for any $f \in I^{s}\left(\dot{\mathcal{L}}^{2, \lambda}\right)$, there exists a sequence of functions $\left(f_{j}\right)_{j \in \mathbf{Z}}$ of $C^{\infty}\left(\mathbf{R}^{n}\right)$ satisfying

$$
\frac{1}{|B|^{\frac{\lambda}{n}}} \sum_{j \geq J} 2^{2 j s}\left\|f_{j}\right\|_{L^{2}(B)}^{2} \leq C\|f\|_{I^{s}\left(\dot{\mathcal{L}}^{2, \lambda}\right)}^{2}
$$

for any ball $B$ of $\mathbf{R}^{n}$ with radius $2^{-J}, J \in \mathbf{Z}$.

Lemma 25. There exists a constant $C>0$ such that for any functions $\left(f_{j}\right)_{j \in \mathbf{Z}}$ with supp $\mathcal{F} f_{j} \subset\left\{\frac{1}{2} 2^{j} \leq|\xi| \leq 2.2^{j}\right\}$, we get

$$
\left\|\sum_{j} f_{j}\right\|_{I^{s}\left(\dot{\mathcal{L}}^{2, \lambda}\right)}^{2} \leq C \sup _{B}\left\{\frac{1}{|B|^{\frac{\lambda}{n}}} \sum_{j \geq J} 2^{2 j s}\left\|f_{j}\right\|_{L^{2}(B)}^{2}\right\}
$$

where the supremum is taken over all balls $B$ of $\mathbf{R}^{n}$ of radius $2^{-J}, J \in \mathbf{Z}$. 
Proof of Lemma 24. Let $f \in I^{s}\left(\dot{\mathcal{L}}^{2, \lambda}\right)$ and $g=I^{-s} f$. If we set $f_{j}=\dot{\Delta}_{j} f$ then $f_{j}=I^{s} \dot{\Delta}_{j} g$.

The proof of Lemma 24 is equivalent to show that

$$
\frac{1}{|B|^{\frac{\lambda}{n}}} \sum_{j \geq J} 2^{2 j s}\left\|I^{s} \dot{\Delta}_{j} g\right\|_{L^{2}(B)}^{2} \leq C\|g\|_{\dot{\mathcal{L}}^{2, \lambda}\left(\mathbf{R}^{n}\right)}^{2}
$$

We decompose $g=g_{1}+g_{2}+g_{3}$ with $g_{1}=m_{2 B} g, g_{2}=\left(g-m_{2 B} g\right) \chi_{2 B}$ and $g_{3}=\left(g-m_{2 B} g\right) \chi_{\mathbf{R}^{n} \backslash 2 B}$ where $B$ is the ball of $\mathbf{R}^{n}$ centered at $x_{0}$ and of radius $2^{-J}, J \in \mathbf{Z}$.

The function $g_{1}$ is constant, $\dot{\Delta}_{j} g_{1}=0$ for any $j$. Then inequality (22) is valid for $g_{1}$.

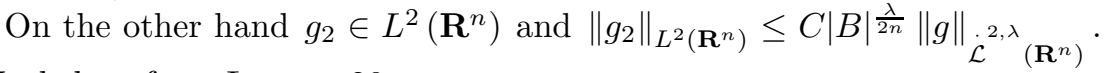
We deduce from Lemma 20

$$
\begin{aligned}
\sum_{j \geq J} 2^{2 j s}\left\|I^{s} \dot{\Delta}_{j} g_{2}\right\|_{L^{2}(B)}^{2} & \leq \sum_{j} 2^{2 j s}\left\|I^{s} \dot{\Delta}_{j} g_{2}\right\|_{L^{2}\left(\mathbf{R}^{n}\right)}^{2} \\
& \leq C \sum_{j}\left\|\dot{\Delta}_{j} g_{2}\right\|_{L^{2}\left(\mathbf{R}^{n}\right)}^{2} \\
& \leq C\left\|g_{2}\right\|_{L^{2}\left(\mathbf{R}^{n}\right)}^{2} \\
& \leq C|B|^{\frac{\lambda}{n}}\|g\|_{\dot{\mathcal{L}}^{2, \lambda}\left(\mathbf{R}^{n}\right)}^{2}
\end{aligned}
$$

Inequality (22) holds for $g_{2}$.

We set $h_{j}=I^{s} \dot{\Delta}_{j} g_{3}, j \in \mathbf{Z}$. We will show that

$$
\left|h_{j}(x)\right| \leq C\|g\|_{\dot{\mathcal{L}}^{2, \lambda}\left(\mathbf{R}^{n}\right)} 2^{J-j-j s}|B|^{\frac{1}{2}\left(\frac{\lambda}{n}-1\right)}
$$

holds for any $x \in B$ and $j \geq J$, and therefore inequality (22) is valid for $g_{3}$.

To show (23) we write

$$
h_{j}(x)=\int_{\mathbf{R}^{n} \backslash 2 B} g_{3}(y)\left(\mathcal{F}^{-1} \psi_{j}^{s}\right)(x-y) d y
$$

where $\psi_{j}^{s}(\xi)=|\xi|^{-s} \theta\left(2^{-j} \xi\right), \theta(\xi)=\varphi(\xi)-\varphi(2 \xi), \varphi$ is the function defining the partition of unity. We have

$$
\left(\mathcal{F}^{-1} \psi_{j}^{s}\right)(x)=2^{j(n-s)}\left(\mathcal{F}^{-1} \psi^{s}\right)\left(2^{j} x\right)
$$

where $\psi^{s}(\xi)=|\xi|^{-s} \theta(\xi)$. 
Since $\psi^{s} \in \mathcal{S}\left(\mathbf{R}^{n}\right)$, for each nonnegative integer $N$ there exists $A_{N}>$ 0 such that

$$
\left|\left(\mathcal{F}^{-1} \psi^{s}\right)(x)\right| \leq A_{N}\left(1+|x|^{2}\right)^{-N}
$$

Now if $x \in B, y \in \mathbf{R}^{n} \backslash 2 B$ and $j \geq J$ then $\left|2^{j}(x-y)\right| \geq 1$ and $\left|x_{0}-y\right| \leq 2|x-y|$. Thus

$$
\begin{aligned}
\left|h_{j}(x)\right| & \leq A_{N} 2^{j(n-s-2 N)} \int_{\mathbf{R}^{n} \backslash 2 B}\left|g_{3}(y)\right||x-y|^{-2 N} d y \\
& \leq A_{N} 2^{j(n-s-2 N)} \sum_{l=1}^{+\infty} \int_{2^{l+1} B \backslash 2^{l} B}\left|g_{3}(y)\right|\left|x_{0}-y\right|^{-2 N} d y \\
& \leq A_{N} 2^{j(n-s-2 N)} \sum_{l=1}^{+\infty} 2^{-2 N(l-J)} m_{2^{l+1} B}\left(\left|g_{3}\right|\right)\left|2^{l+1} B\right| \\
& \leq A_{N} 2^{j(n-s-2 N)} \sum_{l=1}^{+\infty} 2^{-2 N(l-J)} 2^{(l+1-J) n} m_{2^{l+1} B}\left(\left|g-m_{2 B} g\right|\right)
\end{aligned}
$$

Set $\alpha=\left\{\begin{array}{l}0 \text { if } 0 \leq \lambda \leq n \\ 1 \text { if } \lambda>n\end{array}\right.$. Lemma 18 yields

$$
\begin{aligned}
\left|h_{j}(x)\right| \leq & A_{N} 2^{j(n-s-2 N)} \sum_{l=1}^{+\infty} 2^{(n-2 N)(l-J)}\left\{\left|m_{2 B}\left(g-m_{2 B} g\right)\right|\right. \\
& \left.+C(l+1)\left|2^{\alpha l} B\right|^{\frac{1}{2}\left(\frac{\lambda}{n}-1\right)}\left\|g-m_{2 B} g\right\|_{\dot{\mathcal{L}}^{2, \lambda}\left(\mathbf{R}^{n}\right)}\right\} \\
\leq & A_{N} 2^{J-j-j s} 2^{(j-J)(n+1-2 N)}|B|^{\frac{1}{2}\left(\frac{\lambda}{n}-1\right)}\|g\|_{\mathcal{L}^{2, \lambda}\left(\mathbf{R}^{n}\right)} \\
& \times \sum_{l=1}^{+\infty} 2^{l\left(n-2 N+\frac{\alpha}{2}(\lambda-n)\right)}(l+1)
\end{aligned}
$$

For $j \geq J$ and $N$ large we obtain inequality (23). The proofs of Lemma 24 and the injection $I^{s}\left(\dot{\mathcal{L}}^{2, \lambda}\right) \hookrightarrow \dot{\mathcal{L}}^{2, \lambda, s}$ are complete.

Proof of Lemma 25. Proposition 8 yields

$$
\left\|\sum_{j} f_{j}\right\|_{I^{s}\left(\dot{\mathcal{L}}^{2, \lambda}\right)}^{2}=\left\|I^{-s} \sum_{j} f_{j}\right\|_{\dot{\mathcal{L}}^{2, \lambda}\left(\mathbf{R}^{n}\right)}^{2} \leq C\left\|\sum_{j} I^{-s} f_{j}\right\|_{\dot{\mathcal{L}}^{2, \lambda, 0}\left(\mathbf{R}^{n}\right)}^{2}
$$




$$
\begin{aligned}
& \leq \sup _{B} \frac{1}{|B|^{\frac{\lambda}{n}}} \sum_{k \geq J}\left\|\sum_{j} I^{-s} \dot{\Delta}_{k} f_{j}\right\|_{L^{2}(B)}^{2} \\
& \leq C \sup _{B} \frac{1}{|B|^{\frac{\lambda}{n}}} \sum_{k \geq J} \sum_{j=k-1}^{k+1}\left\|I^{-s} \dot{\Delta}_{k} f_{j}\right\|_{L^{2}(B)}^{2}
\end{aligned}
$$

The supremum is taken over all balls $B$ of $\mathbf{R}^{n}$ of radius $2^{-J}, J \in \mathbf{Z}$.

Let $\phi \in C_{0}^{\infty}\left(\mathbf{R}^{n}\right), \phi=1$ on $2 B$ and supp $\phi \subset 3 B$.

Lemma 20 gives for any $j \in \mathbf{Z}$

$$
\begin{aligned}
\left\|I^{-s} \dot{\Delta}_{j} f_{j}\right\|_{L^{2}(B)} & =\left\|\phi I^{-s} \dot{\Delta}_{j} f_{j}\right\|_{L^{2}(B)} \\
& \leq\left\|I^{-s} \dot{\Delta}_{j} \phi f_{j}\right\|_{L^{2}(B)}+\left\|\left[I^{-s} \dot{\Delta}_{j}, \phi\right] f_{j}\right\|_{L^{2}(B)} \\
& \leq C 2^{j s}\left\|\phi f_{j}\right\|_{L^{2}\left(\mathbf{R}^{n}\right)}+\left\|\left[I^{-s} \dot{\Delta}_{j}, \phi\right] f_{j}\right\|_{L^{2}(B)} \\
& \leq C 2^{j s}\left\|f_{j}\right\|_{L^{2}(3 B)}+\left\|\left[I^{-s} \dot{\Delta}_{j}, \phi\right] f_{j}\right\|_{L^{2}(B)}
\end{aligned}
$$

On the other hand

$$
\begin{aligned}
I^{-s} \dot{\Delta}_{j} f_{j}(x) & =\int_{\mathbf{R}^{n}} f_{j}(y)\left(\mathcal{F}^{-1} \psi_{j}^{-s}\right)(x-y) d y \\
& =2^{j(n+s)} \int_{\mathbf{R}^{n}} f_{j}(y)\left(\mathcal{F}^{-1} \psi^{-s}\right)\left(2^{j}(x-y)\right) d y
\end{aligned}
$$

where $\psi_{j}^{-s}(\xi)$ and $\psi^{-s}$ are defined in (24). Therefore, if $x \in B$

$$
\begin{aligned}
{\left[I^{-s} \dot{\Delta}_{j}, \phi\right] f_{j}(x)=} & -2^{j(n+s)} \int_{\mathbf{R}^{n} \backslash 2 B} f_{j}(y)\left(\mathcal{F}^{-1} \psi^{-s}\right)\left(2^{j}(x-y)\right) d y \\
& +2^{j(n+s)} \int_{3 B \backslash 2 B} \phi(y) f_{j}(y)\left(\mathcal{F}^{-1} \psi^{-s}\right)\left(2^{j}(x-y)\right) d y
\end{aligned}
$$

We decompose $\mathbf{R}^{n} \backslash 2 B=\bigcup_{\nu \geq-J+1} F_{\nu}$ with $F_{\nu}=\left\{y \in \mathbf{R}^{n}: 2^{\nu} \leq\left|x_{0}-y\right|\right.$ $\left.\leq 2^{\nu+1}\right\}$. For $\nu \geq-J+1$, if $x \in B$ and $y \in F_{\nu}$, then $|x-y| \sim\left|x_{0}-y\right| \sim 2^{\nu}$. With the aid of inequality (25) we obtain

$$
\begin{gathered}
\left|-2^{j(n+s)} \int_{\mathbf{R}^{n} \backslash 2 B} f_{j}(y)\left(\mathcal{F}^{-1} \psi^{-s}\right)\left(2^{j}(x-y)\right) d y\right| \\
\leq A_{N} 2^{j(n+s)} \sum_{\nu \geq-J+1} \int_{F_{\nu}} 2^{-2 j N}\left|f_{j}(y)\right||x-y|^{-2 N} d y
\end{gathered}
$$




$$
\begin{aligned}
& \leq A_{N} 2^{j(n+s-2 N)} \sum_{\nu \geq-J+1} 2^{-2 \nu N} \int_{F_{\nu}}\left|f_{j}(y)\right| d y \\
& \leq A_{N} 2^{j(n+s-2 N)} \sum_{\nu \geq-J+1} 2^{-2 \nu N}\left\|f_{j}\right\|_{L^{2}\left(F_{\nu}\right)}\left|F_{\nu}\right|^{\frac{1}{2}}
\end{aligned}
$$

For $x \in B$ and $y \in 3 B \backslash 2 B,|x-y| \sim 2^{-J}$, so for any integer $N$, there exists $C_{N}>0$ such that $\left|\left(\mathcal{F}^{-1} \psi^{-s}\right)\left(2^{j}(x-y)\right)\right| \leq C_{M} 2^{-(j-J) 2 N}$. Thus

$$
\begin{aligned}
& \left|2^{j(n+s)} \int_{3 B \backslash 2 B} \phi(y) f_{j}(y)\left(\mathcal{F}^{-1} \psi^{-s}\right)\left(2^{j}(x-y)\right) d y\right| \\
& \quad \leq \quad C_{N} 2^{j(n+s)} 2^{-(j-J) 2 N} \int_{3 B \backslash 2 B}\left|f_{j}(y)\right| d y \\
& \quad \leq \quad C_{N} 2^{j(n+s-2 N)} 2^{-2(-J+1) N}\left\|f_{j}\right\|_{L^{2}\left(F_{-J+1}\right)}\left|F_{-J+1}\right|^{\frac{1}{2}}
\end{aligned}
$$

Hence, for any integer $N$ there exists $C_{N}>0$ such that

$$
\left|\left[I^{-s} \dot{\Delta}_{j}, \phi\right] f_{j}(x)\right| \leq C_{N} 2^{j(n+s-2 N)} \sum_{\nu \geq-J+1} 2^{-2 \nu N}\left\|f_{j}\right\|_{L^{2}\left(F_{\nu}\right)}\left|F_{\nu}\right|^{\frac{1}{2}}
$$

Therefore

$$
\begin{aligned}
\left\|\left[I^{-s} \dot{\Delta}_{j}, \phi\right] f_{j}\right\|_{L^{2}(B)} \leq & C_{N} 2^{j(n-2 N)} 2^{j s}|B|^{\frac{1}{2}} \\
& \times \sum_{\nu \geq-J+1} 2^{\nu\left(-2 N+\frac{\lambda+n}{2}\right)} \frac{1}{\left|F_{\nu}\right|^{\frac{\lambda}{2 n}}}\left\|f_{j}\right\|_{L^{2}\left(F_{\nu}\right)} \\
\leq & C_{N} 2^{(j-J)(n-2 N)} 2^{j s} 2^{-J \frac{\lambda}{2}} \sum_{\nu \geq 1} 2^{\nu\left(-2 N+\frac{\lambda+n}{2}\right)} \\
& \times \frac{1}{\left|F_{\nu-J}\right|^{\frac{\lambda}{2 n}}}\left\|f_{j}\right\|_{L^{2}\left(F_{\nu-J}\right)}
\end{aligned}
$$

We choose $N$ large and we use inequalities (26) and (27) together with Lemma 14 to deduce

$$
\begin{aligned}
& \frac{1}{|B|^{\frac{\lambda}{n}}} \sum_{j \geq J k=j-1} \sum^{j+1}\left\|I^{-s} \dot{\Delta}_{j} f_{k}\right\|_{L^{2}(B)}^{2} \\
& \leq \quad C \frac{1}{|B|^{\frac{\lambda}{n}}} \sum_{j \geq J} 2^{2 j s}\left\|f_{j}\right\|_{L^{2}(3 B)}^{2}+\sum_{j \geq J} C_{N} 2^{2(j-J)(n-2 N)} 2^{2 j s} \\
& \quad \times\left\{\sum_{\nu \geq 1} 2^{\nu\left(-2 N+\frac{\lambda+n}{2}\right)} \frac{1}{\left|F_{\nu-J}\right|^{\frac{\lambda}{2 n}}}\left\|f_{j}\right\|_{L^{2}\left(F_{\nu-J}\right)}\right\}^{2}
\end{aligned}
$$




$$
\begin{aligned}
\leq & C \frac{1}{|B|^{\frac{\lambda}{n}}} \sum_{j \geq J} 2^{2 j s}\left\|f_{j}\right\|_{L^{2}(3 B)}^{2} \\
& +C_{N} \sum_{j \geq J}\left\{\sum_{\nu \geq 1} 2^{\nu\left(-2 N+\frac{\lambda+n}{2}\right)} \frac{2^{j s}}{\left|F_{\nu-J}\right|^{\frac{\lambda}{2 n}}}\left\|f_{j}\right\|_{L^{2}\left(F_{\nu-J}\right)}\right\}^{2} \\
\leq & C \frac{1}{|B|^{\frac{\lambda}{n}}} \sum_{j \geq J} 2^{2 j s}\left\|f_{j}\right\|_{L^{2}(3 B)}^{2}+C_{N}^{\prime} \sup _{\nu \geq 1} \frac{1}{\left|F_{\nu-J}\right|^{\frac{\lambda}{n}}} \\
& \times \sum_{j \geq J-\nu-1} 2^{2 j s}\left\|f_{j}\right\|_{L^{2}\left(F_{\nu-J}\right)}^{2}
\end{aligned}
$$

Finally

$$
\frac{1}{|B|^{\frac{\lambda}{n}}} \sum_{j \geq J} \sum_{k=j-1}^{j+1}\left\|I^{-s} \dot{\Delta}_{j} f_{k}\right\|_{L^{2}(B)}^{2} \leq C \sup _{B} \frac{1}{|B|^{\frac{\lambda}{n}}} \sum_{j \geq J} 2^{2 j s}\left\|f_{j}\right\|_{L^{2}(B)}^{2}
$$

The proof of Lemma 25 is complete.

Proposition 26. Let $s \in \mathbf{R}, 0 \leq \lambda<n+p$ and $2 \leq p<+\infty$. The space $I^{s}\left(\dot{\mathcal{L}}^{p, \lambda}\right)$ is continuously embedded in the space $\dot{\mathcal{L}}^{p, \lambda, s}\left(\mathbf{R}^{n}\right)$.

Proof. Let $f \in I^{s}\left(\dot{\mathcal{L}}^{p, \lambda}\right)$. If we set $g=I^{-s} f$ and $f_{j}=\dot{\Delta}_{j} f$, then $f_{j}=I^{s} \dot{\Delta}_{j} g$. We show that

$$
\frac{1}{|B|^{\frac{\lambda}{n}}} \sum_{j \geq J} 2^{p j s}\left\|I^{s} \dot{\Delta}_{j} g\right\|_{L^{p}(B)}^{p} \leq C\|g\|_{\mathcal{L}^{p, \lambda}}^{p}\left(\mathbf{R}^{n}\right)
$$

As for Lemma 24 we decompose $g=g_{1}+g_{2}+g_{3}$ with $g_{1}=m_{2 B} g$, $g_{2}=\left(g-m_{2 B} g\right) \chi_{2 B}$ and $g_{3}=\left(g-m_{2 B} g\right) \chi_{\mathbf{R}^{n} \backslash 2 B}$ where $B$ is a ball of $\mathbf{R}^{n}$ of radius $2^{-J}, J \in \mathbf{Z}$.

The function $g_{1}$ is constant, $\dot{\Delta}_{j} g_{1}=0$ for any $j$. Then inequality (28) is valid for $g_{1}$. On the other hand $g_{2} \in L^{p}\left(\mathbf{R}^{n}\right)$ and $\left\|g_{2}\right\|_{L^{p}\left(\mathbf{R}^{n}\right)} \leq$

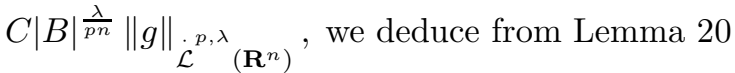

$$
\begin{aligned}
\sum_{j \geq J} 2^{p j s}\left\|I^{s} \dot{\Delta}_{j} g_{2}\right\|_{L^{p}(B)}^{p} & \leq \sum_{j \in \mathbf{Z}} 2^{p j s}\left\|I^{s} \dot{\Delta}_{j} g_{2}\right\|_{L^{p}\left(\mathbf{R}^{n}\right)}^{p} \\
& \leq C \sum_{j \in \mathbf{Z}}\left\|\dot{\Delta}_{j} g_{2}\right\|_{L^{p}\left(\mathbf{R}^{n}\right)}^{p} \\
& \leq C\left\|g_{2}\right\|_{\dot{B}_{p, p}^{0}\left(\mathbf{R}^{n}\right)}^{p} \\
& \leq C\left\|g_{2}\right\|_{L^{p}\left(\mathbf{R}^{n}\right)}^{p}(p \geq 2)
\end{aligned}
$$




$$
\leq C|B|^{\frac{\lambda}{n}}\|g\|_{\dot{L}^{p, \lambda}}^{p}\left(\mathbf{R}^{n}\right)
$$

Inequality (28) holds for $g_{2}$.

Setting $h_{j}=I^{s} \dot{\Delta}_{j} g_{3}$, we show in the same way as for inequality (23) that

$$
\left|h_{j}(x)\right| \leq C\|g\|_{\mathcal{L}_{\left(\mathbf{R}^{n}\right)}^{p, \lambda}} 2^{J-j-j s}|B|^{\frac{1}{p}\left(\frac{\lambda}{n}-1\right)}
$$

holds for any $x \in B$ and $j \geq J$. Therefore inequality (28) holds for $g_{3}$.

Acknowledgements. I am grateful to Professors Pascal Auscher, Gerard Bourdaud and Jacques Camus for their valuable comments and suggestions.

\section{References}

[1] G. Bourdaud, Analyse fonctionnelle dans l'espace euclidien, Publ. Math. Univ. Paris VII, 23 (1987), Réédité, 1995.

[2] J. Bergh and J. Löfström, Interpolation Spaces, Springer Verlag, 1976.

[3] S. Campanato, Proprietà di Hölderianità di alcune classi di funzioni, Ann. Scuola Norm. Sup. Pisa, 17 (1963), 175-188.

[4] S. Campanato, Proprietà di una famiglia di spazi funzionali, Ann. Scuola Norm. Sup. Pisa, 18 (1964), 137-160.

[5] A. El Baraka, Optimal BMO and $\mathcal{L}^{p, \lambda}$ estimates near the boundary for solutions of a class of degenerate elliptic problems, Proc. Conf. Fez (Morocco, 1999), Lecture Notes Pure Appl. Math., 229, New York, Marcel Dekker (2002), 183-193.

[6] A. El Baraka, An embedding theorem for Campanato spaces, Electron. J. Diff. Eqns., 2002 (66) (2002), 1-17.

[7] A. El Baraka, Function spaces of BMO and Campanato type, Electron. J. Diff. Eqns., Conference 09, 2002 (2002), 109-115.

[8] A. El Baraka, Estimates near the boundary for solutions of PDE and interpolation inequalities, Ann. Sci. Math. Québec, 27 (1) (2003), 1345.

[9] A. El Baraka, Optimal BMO and $\mathcal{L}^{p, \lambda}$ estimates for solutions of elliptic boundary value problems, Arabian J. Sci. Engeneering, 30 (1A) (2005).

[10] C. Feffermann and E. M. Stein, $H^{p}$ spaces of several variables, Acta Math., 129 (1972), 167-193. 
[11] M. Giaquinta, Introduction to Regularity Theory for Nonlinear Elliptic Systems, Birkhäuser, 1993.

[12] F. John and L. Nirenberg, On functions of bounded mean oscillations, Comm. Pure Appl. Math., 14 (1961), 415-426.

[13] E. S. Stein and A. Zygmund, Boundedness of translation invariant operators on Hölder and $L^{p}$ spaces, Ann. of Math., 85 (1967), 337349.

[14] R. S. Strichartz, Bounded mean oscillation and Sobolev spaces, Indiana Univ. Math. J., 29 (1980), 539-558.

[15] H. Triebel, Theory of Function Spaces, Birkhäuser, 1983.

[16] H. Triebel, Theory of Function Spaces II, Birkhäuser, 1992.

[17] H. Wallin, New and old function spaces, Proc. Conf. Lund 1986, Lect. Notes Math., 1302, Berlin, Springer (1988), 97-114.

University Mohamed Ben Abdellah

FST Fez

Department of mathematics

BP 2202, Route Immouzer

$\mathrm{Fez}$ Morocco

(E-mail : aelbaraka@yahoo.com) 


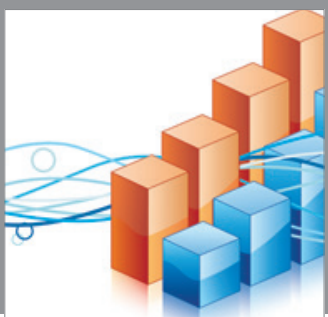

Advances in

Operations Research

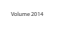

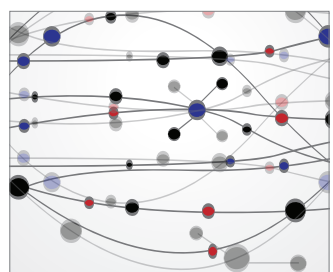

\section{The Scientific} World Journal
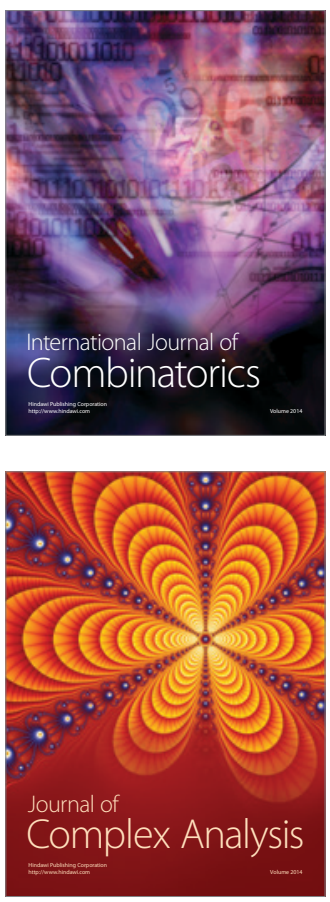

International Journal of

Mathematics and

Mathematical

Sciences
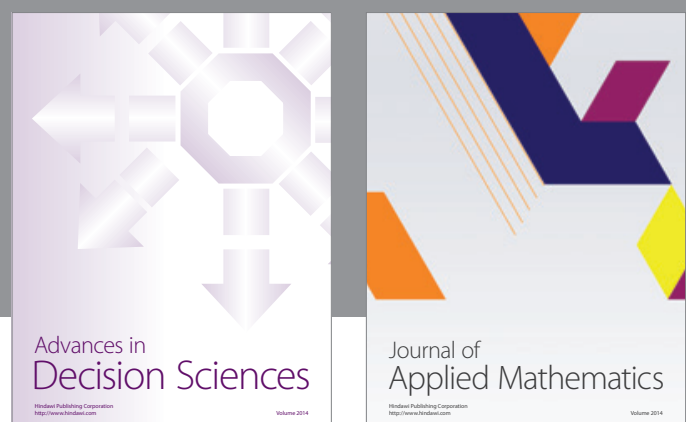

Journal of

Applied Mathematics
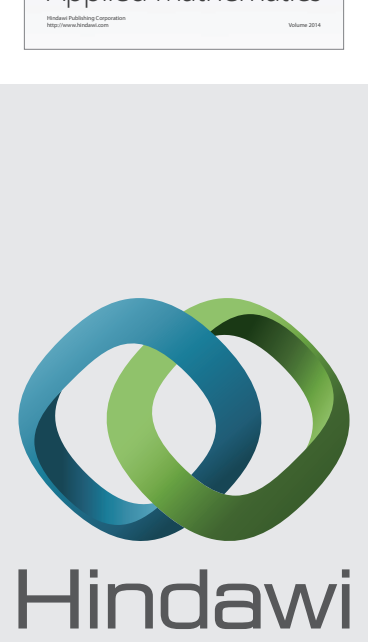

Submit your manuscripts at http://www.hindawi.com
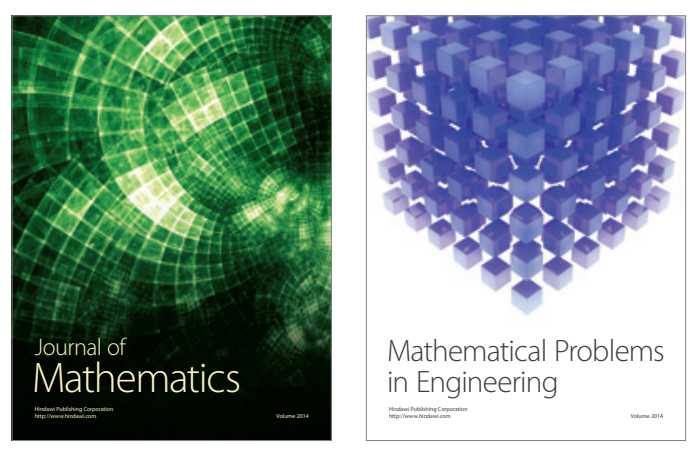

Mathematical Problems in Engineering
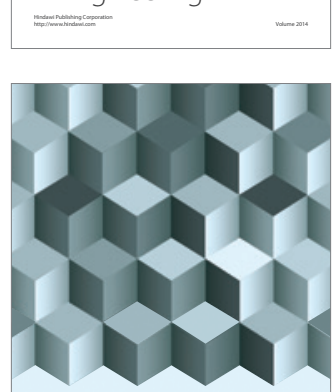

Journal of

Function Spaces
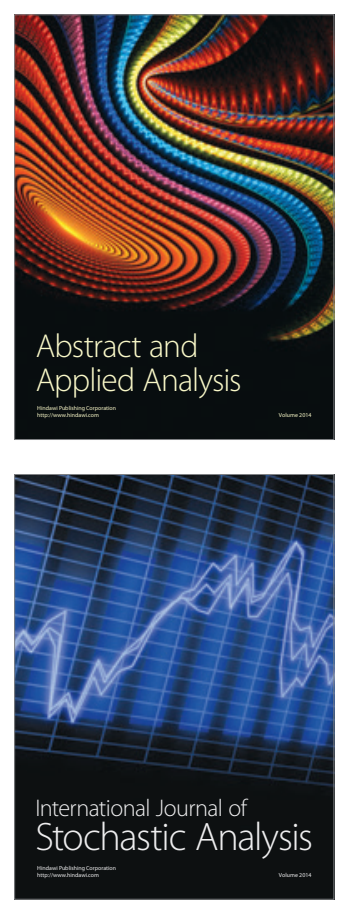

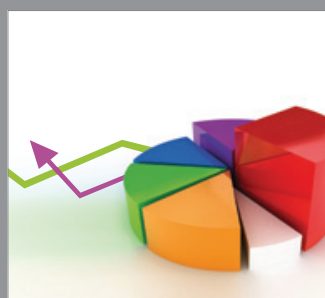

ournal of

Probability and Statistics

Promensencen
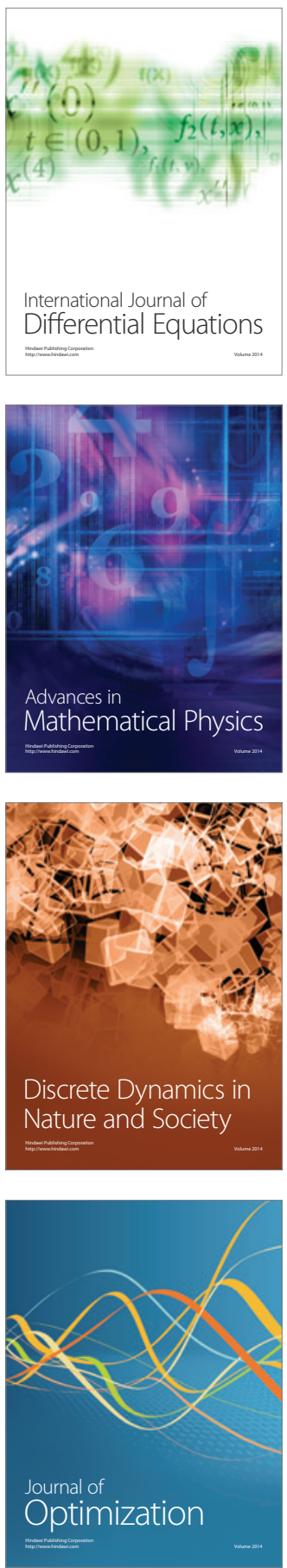